\title{
XIXème Congrès de la Société d'Andrologie De Langue Française
}

\author{
Genève \\ 12 - 13 - 14 Décembre 2002
}

\section{Aristote (384-322 av. J.-C) : \\ Biologiste de la reproduction avant la lettre}

\section{G. ANDROUTSOS}

Histoire de la Médecine, Université d'loannina, Grèce

Aristote est né à Stagyre, en Macédoine Grecque. Du fait qu' une grande partie de son œuvre traite de la génération, Aristote est considéré comme le premier « biologiste de la reproduction ». Il a avancé une théorie selon laquelle la femme tient une place tout à fait mineure dans la conception. Pour Aristote, l'embryon est formé par la seule semence de l'homme qui agit sur une matière constituée, quant à elle, par les menstrues de la femme. Il récuse la théorie d'Hippocrate qui fait intervenir la semence féminine dans la conception. Le mâlc, selon Aristote, apporte par son sperme le principe efficient, le facteur de mouvement, la forme, l'idée, tandis que la femme n'apporte que la matière utile à former l'embryon. Selon Aristote, le sperme est formé à partir du sang le plus pur, et l'ultime produit obtenu est fait d'un mélange d'eau et de pneuma (le souffle) apporté par les artères spermatiques. Il soutient que la force du sperme détcrmine la ressemblance aux parents. Il soutient qu'un sperme qui « domine complètement la matière maternelle » donnera naissance non seulement à un garçon mais qu'en plus il aura tous les caractères de son père. Mais si le père vainqueur en tant que mâle est vaincu en tant que père, il naîtra un garçon qui ressemblera à sa mère...Les ressemblances peuvent provenir aussi de parents éloignés, pensait Aristote. Son explication est que si le mouvement de la semence du père est affaibli, c'est la force de la semence du grandpère qui devient dominante. Aristote a évoqué l'hérédité de l'acquis : les parents porteurs d'une anomalie peuvent la transmettre à leurs enfants, mais « en général, les enfants de parents mutilés naissent avec tous leurs membres...». Aristote pense que la détermination du sexe de l'embryon est établie dès la conception, le mâle est donc l'être normal, parfaitement réussi, tandis que la femme est une « sorte de monstruosité nécessaire au maintient de l'espèce ». Il récuse, par conséquent, l'opinion d'Hippocrate concernant l'influence sur le sexe du côté occupé dans la matrice. Anaxagore prétendait que le testicule droit servait à produire des garçons et que le gauche produisait des filles. Il n'en est rien pour Aristote puisque la semence provient du sang et non des testicules. Il énumère aussi d'autres facteurs susceptibles d'agir sur le sexe de l'enfant à venir, l'âge parental en particulier. Aristote est le premier à affirmer que le développement de l'embryon est progressif, débutant à partir de substances amorphes ce qui annonce la théorie de l'épigénèse. Cette thèse s'opposera violemment à la théorie de la préformation, fondée sur l'idée que le développement de l'embryon résulte de la simple croissance d'un être déjà présent mais minuscule, « invisible ». Les écrits d'Aristote serviront de repère au savoir scientifique sur la génération jusqu'au XVIIe siècle malgré leurs contradictions.

\section{Cancer de la verge : historique du traitement chirurgical}

\section{G. ANDROUTSOS}

Histoire de la Médecine, Université d'loannina, Grèce 
Dès le XVIe siècle Fabrice d'Acquapendente et Ambroise Paré auraient pratiqué déjà des amputations de la verge. En 1635 Scultet rapporte une amputation. En Allemagne Doebel importe l'amputation du pénis au couteau vers la fin du XVII s. La crainte des hémorragies fit découvrir à Ruysch le procédé de la ligature en 1691 . Cette méthode resta classique et ne commença à perdre du terrain que vers la fin du $X V I I I e$ siècle. Les dernières relations de ligature furent faites par Groefe et Binet en 1828. L'amputation de la verge fut néanmoins réhabilitée en partie par Le Dran en 1730 . Ce procédé ne fut repris que timidement, car l'hémorragie était le principal obstacle à la diffusion de la méthode sanglante. Warner (1850) ne pratique l'amputation qu'après avoir placé un tourniquet à la racine de la verge et Pallucci propose la compression des artères sur les branches du pubis. A la fin du XVIIIe siècle les travaux de Hey et de Boyer consacrent définitivement l'emploi du bistouri dans le traitement du cancer de la verge. Depuis le début du XIXe siècle cette méthode est à peu près la seule cn usage. Cependant, d'autres procédés étaient proposés : cautérisation à la pâte caustique de Vienne et au chlorure de zinc, soit au thermocautère. Middeldorpf, le premier, recommande en 1854 l'amputation avec le couteau du galvanocautère, procédé employé en France seulement en 1869 par Sédillot, puis par Amussat (1870), Velpeau, Chassaignac, Trélat, Morel-Lavallée et Ollier. Les avantages sont : absence d'hémorragie, de retention d'urine ou dc pyohémie. Toutefois l'amputation à l'aide d'instrument tranchant dominait ; jusqu'en 1830 on s'était borné à la simple amputation. Delpech faisait l'amputation totale au ras du pubis. L'extirpation totale de la verge avec ablation complète des corps caverneux est pratiquée par Cabadé d'Agen en 1873. Cette opération est perfectionnée par Gould. Vers 1886, Harisson, Horteloup et Montaz ont l'idée de créer, dans la région bulbaire de l'urèthre, en arrière de la cicatrice opératoire, une ouverture, évitant ainsi à la plaie d'être souillée par l'urine. A côté de ces méthodes s'en place une plus radicale : l'émasculation totale. Elle aurait été employée pour la première fois par Annandale en 1873. En 1887, Péan fait une operation se rapprochant de l'émasculation totale : il pratique, d'ailleurs au thermocautère une amputation totale avec castration unilatérale. Ce fut Chalot qui fit la première émasculation totale en France en 1892. Suivi dans cette voie par Albarran, Tédenat, Legueu, Forgue, il en décrivit la technique. Pantaloni publie en 1898 trois cas opérés par lui et Jonnesco, trois cas égalcment en 1901. Serra, dans sa thèse de 1903 , réunit une vingtaine d'observations. Depuis, l'émasculation totale ne l'est plus aussi systématiquement à l'heure actuelle. Küttner a montré par ses recherches qu'il n'y a pas d'avantage à enlever les bourses et les testicules sains, la récidive, après amputation du pénis cancéreux ne survenant qu'au niveau des ganglions inguinaux et non dans les bourses ou sur les cordons spermatiques. C'est d'ailleurs la conclusion à laquelle aboutit Legueu en 1908. Actuellement, à côté du traitement chirurgical qui s'applique à la majorité des cas rencontrés en clinique, nous devons signaler la curiethérapie ainsi que la chimiothérapie qui ont leurs indications particulières.

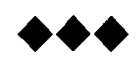

\section{" L'eau fondante de Guilbert de}

Preval " : le premier preservatif chimique

\section{G. ANDROUTSOS}

Histoire de la Médecine, Université d'loannina, Grèce

"L'eau fondante de Guilbert de Préval " était un préservatif chimique des maladies vénériennes recommandé à la fin du XVIlle siècle par Guilbert de Préval qui était le médecin de "la Gourdan ", personne connue pour tenir une des maisons de rendez-vous les plus célèbres de Paris. Son mode d'emploi est rapporté par le docteur Baude (thèse de médecine de Paris, 1927). "Quiconque voudra se servir de cette eau prenne un gobelet ou un autre vase fait non d'aucun métal mais de terre ou de verre, qu'il y plonge la partie qu'il voudra rendre invulnérable au virus vénérien, qu'il l'en abreuve avec soin et à plusieurs reprises lorsqu'il se sera exposé au commerce d'une femme suspecte ou décidément gâtée. Quant aux femmes, qu'elles s'en fassent des injections fréquentes et profondes ". L' " eau fondante " n'aurait été qu'un mélange d'eaux de chaux, de sel marin, d'une très petite quantité de mercure et d'un «principe odorant». Son efficacité semble avoir été douteuse. tout au moins pour le fils du duc de Duras, si l'on en croit Bachaumont. L'inventeur de l' " eau fondante, Claude-Thomas-Guillaume Guilbert de Préval, n'était pas un charlatan mais un honorable docteur-régent de la Faculté de Médecine de Paris (1759) spécialisé en syphiligraphie. Ami des écrivains Nicolas Restif de la Bretonne (1734-1806) et LouisSébastien Mercier (1740-1814), il semble avoir été en froid avec ses confrères comme d'ailleurs la plupart des médecins qui soignaient les vénériens, ces traitements étant plus généralement du ressort des chirurgiens et des empiriques. Sur demande de M. de Saint-Laurent, Préval " muni de son eau affronta la syphilis " à plusieurs reprises, en 1771, dans une petite maison de Popincourt, devant le duc de Chartres et le prince de Condé. "L'expérimentation » ayant été concluante, M. de Sartine, alors Lieutenant général de la police, s'intéressa à cette eau et entreprit de son côté de faire faire des essais sous le contrôle du doyen et de trois docteurs de la Faculté. Préval 
vendait fort bien son eau et prétendait même avoir prémuni 2700 soldats et 6200 bourgeois de Paris ! Mais en juillet 1772, une réclame imprimée vantant les mérites de l'eau fondante sera déposée sur le bureau de Le Thieullier, doyen de la Faculté de médecine, laquelle Faculté avait déjà été indisposée par les " expérimentations » de Préval. Un énorme scandale éclatera, qui durera plusieurs années. Préval, après avoir été tout d'abord interdit d'assistance « aux assemblées et actes publiques » de la Faculté, finira par perdre son titre de docteur-régent, sera définitivement exclu de la Faculté et ruiné (1776).

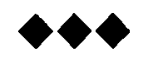

\section{L'Eglise et le contrôle des naissances}

\section{G. ANDROUTSOS}

Histoire de la Médecine, Université d'loannina, Grèce

L'Eglise chrétienne depuis ses débuts était hostile à la contraception. 57 apr. J.-C. : Paul stigmatise les mœurs « contre nature » des païens ; 80 : Propos de Jesus sur les eunuques volontaires (L'Evangile selon saint Matthieu); 95 : L'Apocalypse de Jean condamne les «pharmakoi »; 98: La Didaché attaque les « tueurs de descendance "; 111 : Pline le Jeune dénonce les «vices " des « chrétiens »;177: Athénagoras affirme que les chrétiens ne s'unissent que pour procréer et s'abstiennent pendant la grossesse ; 199 : Marcus Minucius Felix dénonce comme parricides les femmes qui par des drogues «éteignent la naissance d'un homme futur »; 190 : Saint Clément interdit de gaspiller la semence ; 220 : Les chrétiens tiennent l'usage des drogues contraceptives pour un meurtre ; 300 : Lactance assimile les sodomites à des parricides ; $374: \mathrm{La}$ loi tient les infanticides pour des homicides ; 382 : Saint Jérôme dénonce les pratiques contraceptives des jeunes filles de Rome et donne sa forme anti-contraceptive à l'histoire d'Onan ; 390 : Décret de Valentinien vouant au feu les sodomites ; Saint Jean Chrysostome condamne les rapports avec les prostituées parce qu'ils impliquent la contraception ; IVe s. : Saint Augustin stigmatise les procédés contraceptifs et la continence périodique ; 503 : Césaire assimile l'usage des drogues stérilisantes à une multitude d'homicides ; 572 : Saint Martin fait adopter par le concile de Braga un canon contre les drogues stérilisantes ; XIe s. : Gratien intègre dans son Décret deux canons relatifs à la contraception ; 1248 : Albert le Grand affirme que les rapports conjugaux pendant la grossesse ne constituent pas un péché mortel ; 1515 : Sylvestre condamne l'étreinte réservée et le refus du devoir conjugal pour limiter le nombre des enfants ; 1588 : La bulle Effraenatam voue à l'excom- munication et à la mort ceux qui procurent ou utilisent des «poisons de stérilité»; 1602 : Sanchez justifie l'acte conjugal sans souci de l'intention des conjoints tant qu'ils ne font rien pour éviter la conception. 11 suggère l'interruption du coït fornicateur et autorise les femmes violées à expulser la semence reçue. Il oblige l'épouse d'un onaniste à rendre quand même le dû conjugal ; 1798 : Malthus publie An Essay on Principle of Population; 1853 : Le Saint Office condamne toute forme de coopération losque le mari use d'un préservatif. ; 1878 : La Pénitencerie recommande l'interrogatoire en cas de soupçons fondés d'onanisme ; 1909 : Les évêques belges envoient à leurs prêtres une Instruction sur l'onanisme qui prescrit la lutte à outrance ; 1916 : Si le mari utilise un préservatif, l'épouse devra lui résister comme à un violateur, dit la Pénitencerie ; 1920 : Les évêques allemands prescrivent aux maris d'arracher les instruments contraceptifs dont voudraient se servir leurs femmes ; 1930 : L'Eglise anglicane admet la contraception pour des motifs sérieux ; 1951 : Pie XII estime « naturelle » la recherche du plaisir par les conjoints ; 1958 : Pie II condamne tout emploi de la pilule à des fins directement contraceptives ; 1963 : L'évêque de Bois-le-Duc proclame que le choix des moyens de régulation des naissances fait partie des la responsabilité des conjoints. Les évêques discutent la légitimité des contraceptifs chimiques ; 1964 : L'archevêque de Westminster affirme que la contraception est condamnée par les lois divines et ne saurait être une question ouverte.

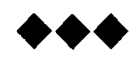

\section{Venette Nicolas (1633-1698) et le premier traité de sexologie}

\section{G. ANDROUTSOS}

Histoire de la Médecine, Université d'loannina, Grèce

Premier « sexologue », Venette, publia son ouvrage capital La Génération de l'homme, ou Tableau de l'amour conjugal (1688). L'utérus est toujours le très avide « aspirateur à sperme " responsable des appétits sexuels des femmes. Le clitoris est bien le siège de la «lascivité " féminine. Les différentes positions coïtales préoccupent Venette. Après quoi il critique les postures debout ou assises, qui procurent une « mauvaise aspersion » de la matrice. Pour préconiser le « bon vieux missionnaire ", mais aussi les positions a tergo, qui préservent le fruit de la femme enceinte. Venette conclut qu'après tout on peut baiser sa femme de 
quelque manière que ce soit, pourvu que l'on ne commette pas de faute contre la propagation des hommes. Bon médecin, il est aussi un des rares à ne pas jeter l'anathème sur le sang menstruel et ne croit pas aux dangers du coït pendant les règles. Les troubles de l'érection paraissent bien compréhensibles à Venette. L'homme se fatigue plus que la femme. C'est lui qui, dans les rapports conjugaux, "agit presque tout seul, tandis que la femme ne fait que souffrir les caresses d'un homme dans une posture aisée ». Venette est quasi le seul à dépeindre les troubles de l'éjaculation. Avec pas moins de sept différents cas parmi lesquels se trouvent l'éjaculation molle et l'éjaculation rétrograde. Les causes de ces empêtrements masculins relèvent de trois grandes rubriques $d$ ' « époque ». 1) la « morale ». On y trouve ressassées les vieilles rengaines sur l'épuisement par la vie dissipée, les excès vénériens, l'abus du plaisir solitaire. 2) celle des sortilèges. Venette ne croit pas qu'un magicien puisse s'opposer aux violents desseins de la nature. 3) celle des causes organiques : Venette connaissait les imperfections anatomiques masculines, l'hypospadias, le phimosis, la brièveté du frein, ainsi que le paraphimosis. II dispute des dimensions fautives du membre viril, trop court, ou trop long, ou trop large. Le chapitre étiologique de l'impuissance organique établi par Venette lui fait honneur. On y trouve en effet : 1) Les causes endocriniennes : insuffisance ou absence des testicules, ectopie, cause certaine de stérilité ; 2) Les causes traumatiques : lésions des nerfs érecteurs ; 3 ) Les causes infectieuses : prostatite chronique ; 4) Les causes toxiques : l'alcoolisme ; 5) Les causes neurologiques : les nerfs qui sortent de l'os sacrum sont «faibles, ou bouchés »; ou l'homme souffre d'une palalysie des membres inférieurs ; 6) La sénescence : le vieillissement artériel ; 7) Les nodosités : maladie de Lapeyronie. Pour les freins trop courts, Venette propose unc section transversalc. Le phimosis doit être opéré par des incisions de chirurgie plastique, comme par les Antiques, et non par la circoncision judéo-islamique. Il recommande aux porteurs de verge trop longue l'usage d'un écusson de cuir, de liège bien cotonné, renouvelé des anneaux antiques, pour ne pas « empaler » le cul-de-sac postérieur de leurs partenaires. Il dénonce les remèdes de bonne femme censés allonger les verges trop courtes, frictions, onguents, emplâtres. Il connaît les aphrodisiaques mais ne leur accorde pas grand crédit. Il met en garde contre leurs dangers, en particulier la néphrite hématurique mortelle provoquée par la cantharide. De même qu'il compte sur le charme naturel des femmes pour combattre les maléfices de sorciers, il s'en remet à la plus classique thérapeutique de couple en matière d'impuissance. Il fait un tableau horrifiant de l'hypertrophie clitoridienne. Il se résigne à préconiser l'abstention chirurgicale.

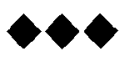

Bilan de quatre ans d'assistance médicale à la procréation avec sperme de donneur

\author{
V. ACHARD ${ }^{1}$, O. PAULMYER-LACROIX ${ }^{1}$, J. SAIAS- \\ MAGNAN ${ }^{1,2}$, C. GUILLEMAIN ${ }^{3}$, A. NOIZET $^{3}$, \\ J. M. GRILLO 1,3 \\ ${ }^{1}$ AP-HM Conception, Laboratoire de Biologie de la \\ Reproduction, 147 bd Baille, 13385 Marseille \\ cedex 05. \\ 2AP-HM Conception, Centre de Procréation Médica- \\ lement Assistée, 147 bd Baille, 13385 Marseille \\ cedex 05. \\ ${ }^{3}$ C.E.C.O.S. Hôpital la Conception, 147 bd Baille, \\ 13385 Marseille cedex 05.
}

\section{Introduction :}

L'assistance médicale à la procréation avec sperme de donneur est la plus ancienne solution à l'infertilité masculine et permet de prévenir une transmission de pathologies génétiques ou infectieuses à la descendance.

\section{Matériel et Méthodes :}

Nous avons étudiés rétrospectivement 367 cycles d'insémination (IAD) et 62 cycles de fécondation in vitro (FIVD) avec sperme de donneur effectués dans le centre d'assistance médicale à la procréation de l'Hôpital de la Conception à Marseille (France) entre janvier 1998 et mai 2002. Toutes les inséminations étaient pratiquées le jour de l'ovulation, par voie intra utérine après préparation du sperme. Elles étaient en majorité $(92 \%)$ couplées à une stimulation de l'ovulation. Ces tentatives concernent 122 couples dont le critère d'inclusion était de pratiquer au moins un cycle d'IAD.

\section{Résultats :}

Trente neuf couples ont obtenu au moins une naissance vivante, huit d'entre eux ont désiré un deuxième enfant, obtenu dans cinq cas. Les taux moyens de succès par cycle (TMSC) sont de $21,2 \%$ pour les IAD, et de $30,1 \%$ en FIV-D pour la période concernée. Cinquante deux enfants sont nés en IAD et 19 en FIV-D. Le taux cumulatif de grossesses en IAD est de $50 \%$ à la sixième tentative. Parmi les paramètres étudiés suivants : indication, pathologie féminine associée, durée de l'infertilité, rang de la tentative, stimulation de l'ovulation, technique utilisée pour préparer le sperme, caractéristiques du sperme des donneurs observés lors du test de décongélation et à la fin de la préparation du sperme, âge de la patiente, seuls les deux derniers ont une influence significative sur les TMSC en IAD. Aucun facteur influençant le TMSC en FIV-D n'a pu être déterminé. 


\section{Conclusion :}

Bien que les paramètres du sperme du donneur ainsi que les techniques de préparation du sperme semblent avoir une influence sur le rendement de l'IAD, les paramètres féminins sont les déterminants principaux de la réussite de l'assistance médicale à lá procréation avec sperme de donneur.

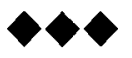

\section{Résultats de l'enquête de la SALF concernant la pratique de la recherche des microdélétions du chromosome $Y$ : biologie moléculaire}

\author{
I. AKNIN-SEIFER1,2, H. LEJEUNE 3, R. LEVY1 \\ 1 Laboratoire de Biologie de la Reproduction et \\ 2 Laboratoire de Génétique de l'Hôpital Nord, \\ Saint-Etienne, 42055 , \\ 3Département de Médecine de la Reproduction, \\ Hôpital E. Herriot, Lyon.
}

\section{Introduction :}

Les microdélétions du chromosome Y peuvent être détectées chez les patients infertiles présentant des troubles sévères de la spermatogenèse. Ce dépistage est conseillé avant réalisation de l'ICSI. Le but de notre enquête était tout d'abord de faire "l'état des lieux » sur la pratique en France du dépistage des microdélétions du chromosome $Y$ en tentant de répondre aux questions suivantes :

- Combien de laboratoires recherchent les microdélétions du chromosome Y ?

- Quclle activité cette analyse représente t-elle?

- Les indications et les paramètres spermatiques sont-ils connus des laboratoires effectuant l'analyse?

- Quelles sont les méthodes employées ?

- Comment est facturée cette analyse?

Les objectifs, à terme, étaient de pouvoir discuter :

- des méthodes les plus adaptées selon le rapport coût efficacité ;

- d'une prise en charge par les organismes d'Assurance Maladie ;

- et de la mise en place d'un « contrôle de qualité* » national.

(* échantillons positifs et négatifs soumis à l'analyse dans les différents laboratoires)
Matériel et Méthodes :

Questionnaire : Un questionnaire spécifique destiné aux biologistes moléculaires a été élaboré. Centre : Les questionnaires ont été adressés aux responsables biologistes des centres agréés en AMP, en France métropolitaine.

\section{Résultats :}

30 laboratoires font cette détection (seuls 29 ont effectivement renvoyé leur formulaire). 29 réponses «Biologistes Moléculaires » ont donc été obtenues soit par retour de questionnaires. soit lors d'un entretien téléphonique. Un total de 10399 analyses a été conduit dans les 29 laboratoires ayant répondu à l'enquête avec une augmentation des examens au cours des années (2617 examens au cours de l'année 2000). Les laboratoires ont connaissance des données cliniques pour $76 \%$ des centres ( 22 sur 29 ) et des données du spermogramme pour $79 \%$ des centres $(23$ sur 29). Les résultats concernant la réalisation de la détection des microdélétions mettent en évidence une extrême diversité des pratiques. La majorité $(66 \%)$ des centres pratiquent une technique « maison » (19 sur 29), deux centres (7\%) utilisent un Kit commercial, et seulement 8 centres (28\%) suivent les recommandations européennes. Cette analyse est facturée en moyenne $606 \mathrm{BHN}$ (de 200 à 2350 BHN).

\section{Conclusion :}

L'activité de recherche des microdélétions est en croissance régulière. Cependant cette analyse suscite encore de nombreuses questions. L'évolution vers une technique standardisée, soumise à un contrôle de qualité national, ainsi que la reconnaissance et la cotation de cette analyse par les organismes d'assurance maladie apparaissent comme des étapes essentielles à sa généralisation en routine.

Résultats de l'enquête de la SALF concernant la prescription de la recherche des microdélétions

du chromosome $Y$ : prescripteurs

\section{AKNIN-SEIFER1,2, H. LEJEUNE 3, R. LEVY1}

1 Laboratoire de Biologie de la Reproduction et

2 Laboratoire de Génétique de l'Hôpital Nord, Saint-Etienne, 42055 ,

3Département de Médecine de la Reproduction, Hôpital E. Herriot, Lyon. 


\section{Introduction :}

La prescription de la recherche des microdélétions du chromosome $\mathrm{Y}$ en cas de troubles sévères de la spermatogenèse est conseillée avant réalisation de l'ICSI. Le but de notre enquête était tout d'abord de faire "l'état des lieux » sur la pratique en France de la prescription des microdélétions du chromosome $\mathrm{Y}$ en tentant de répondre aux questions suivantes :

- Combien de praticiens et de centres d'AMP demandent la recherche des microdélétions du chromosome $\mathrm{Y}$ ?

- Qui sont les praticicns prescripteurs ?

- Quelles sont les indications retenues?

- Comment est financé le dépistage ?

Les objectifs, à terme, ćtaient de pouvoir discuter :

- d'une prise en charge par les organismes d'Assurance Maladie ;

- d'un consensus en ce qui concerne les indications du dépistage.

\section{Matériel et Méthodes :}

Questionnaire : Un questionnaire spécifique destiné aux prescripteurs de cette analyse a été adressé aux responsables cliniciens ou biologistes des 88 centres agréés en AMP.

\section{Résultats :}

72 centres ont répondu soit par retour des questionnaires, soit lors d'un entreticn téléphonique (taux de réponse de $82 \%)$ : 42 centres $(58 \%)$ prescrivent cette détection (25 publics, 16 privés et 1 mixte), 30 centres ( $42 \%$ ) ne le font pas ( 9 publics, 20 privés et 1 mixte). Pour les centres d'AMP ne demandant pas cette analyse, les principales raisons sont : " analyse non disponible localement " $(60 \%)$, « coût élevé de l'analyse » $(43 \%)$ et " ne change pas la prise en charge » $(36 \%)$.

Les prescripteurs sont gynécologues (33\% du total des prescripteurs ayant répondu), urologues $(26 \%)$ et biologistes $(27 \%)$. Les principales indications retenues sont les azoospermies sécrétoires $(54 \%)$ ( 21 centres sur 36$)$. Les azoospermies de toutes origines représentent les $38 \%$ restant ( 15 centres).

À noter, 3 centres ne prescrivent pas du tout la recherche en cas d'azoospermic ( $8 \%$ ). Pour les oligozoospermies, $39 \%$ des prescripteurs retiennent comme seuil 5 Millions de $\mathrm{spz} / \mathrm{ml}$ et autant $(39 \%)$ celui de 1 Million de spz/ml. 8 centres $(21 \%)$ ne retiennent pas les oligozoospermies comme indications. De façon paradoxale, plusieurs centres considèrent la notion d'infertilité familiale comme indication principale de cette recherche. En dehors de protocoles de recherche $(31 \%)$, cette analyse est à la charge du patient (24\%) ou de l'Hôpital (24\%).

\section{Conclusion :}

La prescription de la recherche des microdélétions connaît un essor récent. L'évolution vers un consensus sur les indications est souhaitable. La reconnaissance de cette analyse par lcs organismes d'assurance maladie apparaît comme une étape essentielle à sa généralisation en routine. L'analyse des réponses à ce questionnaire a révélé une forte demande de diffusion d'information sur ce sujet.

\section{Mutations du gène de la polymérase gamma de l'ADN mitochondrial (POLG) associées à l'infer- tilité masculine}

\author{
I. AKNIN-SEIFER1,2, R.L. TOURAINE2, \\ A. COMBES2, J.L. LAURENT1', B. LAURAS2, \\ R. LEVY1
}

1 Laboratoire de Biologie de la Reproduction et 2Senvice de Génétique, Saint-Etienne, 42055, France.

\section{Introduction :}

Le gène POLG localisć en 15q24-15q26 code pour une sous-unité catalytique de l'ADN polymérase mitochondriale, $\gamma$. La partie $\mathrm{N}$ terminale contient une série de 10 glutamines codées par une répétition CAG. Une augmentation du nombre de répétitions de triplets CAG est associée à différentes maladies humaines, comme la chorée de Huntington et certaines formes d'atrophie spinocérébelleuse.

Rovio et al ont comparé le nombre de répétitions des triplets CAG dans une population d'hommes contrôle (population générale), d'hommes fertiles et différentes populations d'hommes infertiles. Il a observé une association entre l'absence de l'allèle normal (correspondant aux 10 répétitions) et l'infertilitć masculine, à l'exclusion de l'azoospermie.

\section{Matériel et Méthodes :}

Patients : 177 patients infertiles et 28 témoins fertiles et normospermiques.

Amplification par $P C R$ : la région des triplets CAG du gène POLG a été amplifiée et l'analyse de fragment réalisée sur un séquenceur Beckman CEQ2000 XL. 


\section{Résultats :}

(1) L'allèle prédominant de 10 répétitions CAG a été retrouvé chez tous les sujets normospermiques et fertiles de façon homozygote ou hétérozygote (génotype 10/10 ou $10 / \mathrm{x})$. (2) Chez les sujets infertiles, dans $2,8 \%$ des cas $(5$ sur 177), on retrouve à l'état homozygote un nombre de répétitions différent de 10 : dans 2 cas, les sujets sont homozygotes pour un allèle mutant, et dans les 3 autres cas, les sujets sont hétérozygotes composites. (3) Les sujets infertiles sont plus souvent hétérozygotes $10 / \mathrm{x}$ ( $25 \%$ ) que les sujets fertiles $(14 \%)$. Les 5 patients homozygotes pour l'absence de l'allèle normal présentent tous une oligozoospermie sévère associée à une asthénozoospermie. Ils représentent $6,1 \%$ des oligozoospermies inférieures à 5 millions de spz/ml $(5,3 \%$ de toutes les oligozoospermies). De plus, ce pourcentage augmente avec la sévérité de l'oligozoospermie (7\% dans les oligozoospermies de moins de 1 million de spz/ml).

\section{Conclusion :}

Nos résultats sont les premiers à confirmer ceux de Rovio et al. Ils indiquent que lorsqu'il n'y a aucune autre étiologie à l'infertilité masculine, POLG contribue à environ $5 \%$ des oligozoospermies. Un dépistage précoce pourrait permettre un conseil génétique approprié puisque cette atteinte génétique est probablement de transmission autosomique récessive. Des études complémentaires sont nécessaires pour mieux comprendre le rôle de cette expansion de polyglutamine dans l'infertilité masculine.

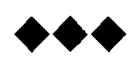

\section{Caractéristiques de morphologie et de mouve- ment de spermatozoïdes de Rattus rattus provenant de trois écosystèmes guadelou- péens : impact probable des pollutions envi- ronnementales}

\section{A. BAZIRE, 2 D. SCHORVAERT, 3 P. JOUANNET, 4 L. MULTIGNER, 3 J. AUGER}

1 Centre Antipoisons de Paris, Hôpital Fernand Widal, 2 Laboratoire d'Analyse d'Images en Pathologie Cellulaire, Hôpital Saint Louis, 3 Laboratoire de Biologie de la Reproduction, CHU Cochin, Paris, 4 INSERMU 435 - GERM, Université de Rennes 1, Rennes

\section{Introduction :}

Dans les Antilles françaises, différentes données récentes suggèrent un impact défavorable des pollutions environnementales sur la reproduction de la faune sauvage. En 2000 a été initié le projet multidisciplinaire ORCHIDEE destiné à étudié plus particulièrement cet impact chez les rats mâles capturés dans trois écosystèmes de la Guadeloupe : un site vierge (A) de toute pollution environnementale situé dans le parc national, un site isolé (B), a priori dépourvu de pollution directe, l'îlet Fajou, et un site agricole $(\mathrm{C}$, bananeraie) a priori fortement exposé notamment à de polluants phytosanitaires.

\section{Matériel et Méthodes :}

Se basant sur des approches de toxicologie expérimentale dans le modèle rat qui indiquent un impact de nombreux composés chimiques sur la formation et la maturation des spermatozoïdes, le présent travail s'est précisément intéressé à deux aspects de la qualité spermatique de l'une des espèces sauvages capturées, le rat noir (Rattus rattus) : la morphologie détaillée des têtes de spermatozoïdes épididymaires et le mouvement de ces spermatozoïdes. Pour ce faire, nous avons mis au point d'une part une méthode d'analyse de la morphométrie et de la densitométrie des têtes de spermatozoïdes par analyse d'image (système SAMBA) en microscopie de fluorescence (x1000, coloration au Hoechst 33342, 150 spermatozoïdes/animal, 21 animaux étudiés), d'autre part, une méthode semi-automatique d'analyse des trajectoires des spermatozoïdes par microvidéographie assistée par ordinateur $(x 67,50 \mathrm{~Hz}$, acquisitions sur ? sec, 60 trajectoires analysées/animal, 42 animaux étudiés). Ces deux méthodes ont permis le calcul de 22 paramètres morphologiques et 11 paramètres de mobilité sur lesquels se sont basées les comparaisons statistiques entre sites.

\section{Résultats :}

1. Analyse morphométrique et densitométrique des têtes des spermatozoïdes.

Il existait des différences significatives entre les 3 sites pour l'ensemble des paramètres étudiés, en dépit d'une variabilité interindividuelle assez importante. Ces différences n'étaient pas expliquées par des différences de poids et d'âge des animaux (âge évalué à partir de la mesure du poids du cristallin). En comparaison au site A, les têtes des spermatozoïdes des animaux des sites $B$ et $C$ (potentiellement exposés) avaient une surface plus petite, une forme moins régulière et une moindre courbure, ceux du site $\mathrm{B}$ avaient une chromatine moins condensée et ceux du site $C$, une chromatine plus immature.

\section{Analyse des caractéristiques du mouvement.}

En dépit d'une variabilité interindividuelle là aussi assez importante, il existait des différences marquées dans la comparaison des animaux des sites A et B, les animaux du site $B$ ayant des caractéristiques de vitesse et de forme de 
trajectoire significativement plus faibles que celles des spermatozoïdes du site vierge. Par contre, les animaux du site $C$ avaient, dans l'ensemble, des caractéristiques de mouvement comparables à celles des animaux du site vierge. Il existait une relation significative et positive entre certaines caractéristiques du mouvement et le poids des animaux (amplitude moyenne de la tête et distance linéaire moyenne parcourue). Il n'a pas été trouvé de relation significative entre les caractéristiques du mouvement et l'âge des animaux.

3. Relation avec les caractéristiques toxicologiques usuelles (âge, poids des organes, réserves spermatiques, dosages hormonaux).

Des corrélations globales ont été mises en évidence entre les caractéristiques de forme des têtes (facteur de forme et rapport corde/arc et la réserve testiculaire), relations avec les valeurs de réserve spermatique non retrouvées au sein de chaque site pris séparément. Par ailleurs, la vitesse curvilinéaire moyenne était inversement corrélée à la réserve spermatique.

Ces données rapportant des différences de la formation (morphologie) mais aussi de la maturation (mobilité) des spermatozoïdes en fonction des sites de capture suggèrent un possible rôle délétère de l'environnement. La moindre qualité à la fois de la mobilité et de la morphologie des spermatozoïdes des animaux capturés sur l'îlet Fajou font évoquer d'éventuels facteurs de pollution indirecte, ce site étant localisé dans le grand cul de sac marin, zone de convergence des effluents des rivières polluées par l'agriculture et les industries de l'île (résidus de pesticides, métaux lourds, etc...). Dans le site bananeraie, site exposé en permanence et directement à de nombreux pesticides, les différences portaient essentiellement sur la morphologie des spermatozoïdes qui apparaissaient globalement plus immatures, suggérant un éventucl impact de ces composés ou de leur métabolites sur la morphogenèse spermatique. Il faut cependant préciser que les conditions d'alimentation dans ces trois écosystèmes pourraient différer de manière notable et ainsi être un facteur également impliqué dans les différences observées. Il est prévu de confronter ultérieurement ces données aux résultats des mesures d'exposition aux pesticides et métaux lourds faites sur les tissus (graisse, os, muscle) prélevés à cet effet.

\section{Relation entre le taux de fragmentation sperma- tique et le taux de grossesse lors de tentatives de Fécondation In Vitro}

\section{Introduction :}

Les caractéristiques spermatiques standard (spermogramme, test de migration-survie, analyse automatisée du mouvement) ne sont pas des prédicteurs fiables pour le résultat d'une tentative de Fécondation in Vitro (FIV). La qualité du génome spermatique joue un rôle important dans l'évolution précoce de l'embryon et dans le succès d'une tentative de FIV. La fragmentation de l'ADN spermatique est un reflet de la qualité du génome spermatique et sa mesure pourrait être un facteur pronostique du résultat d'une tentative de FIV.

\section{Matériel et Méthodes :}

Une étude prospective a été réalisée à l'Hôpital Edouard Herriot de Lyon dans le Département de Médecine et Biologie de la Reproduction. Le taux de fragmentation de l'ADN spermatique d'une cohorte de 104 couples entant dans un programme de Procréation Médicalement Assistée (FIV conventionnelle ou ICSI) a été mesuré par technique TUNEL. Le taux de fragmentation a été corrélé avec les caractéristiques du sperme et les résultats de la tentative de FIV.

\section{Résultats :}

Une corrélation négative a été trouvée entre les caractéristiques spermatique (numération, mobilité, morphologie) du sperme et le taux de fragmentation de l'ADN. Une liaison significative a été trouvée entre le taux de fragmentation de l'ADN spermatique et le taux de fécondation (un taux de fragmentation supérieur à $10 \%$ s'accompagne d'une chute du taux de fécondation). Aucune corrélation n'a été trouvée entre le taux de fragmentation de l'ADN spermatique et la qualité des embryons obtenus. Un taux de fragmentation spermatique ćlevé (supérieur à $15 \%$ ) est un facteur péjoratif pour obtenir une grossesse quand une ICSI est réalisće, ce résultat n'est pas retrouvé lorsqu'une FIV conventionnelle est exécutée. 


\section{Conclusion :}

Le taux de fragmentation de l'ADN spermatique semble être un facteur pronostique pertinent pour le taux de fécondation et l'obtention d'une grossesse lorsqu'une ICSI est réalisée. La qualité des embryon basée sur des critères morphologiques ne semble pas corrélée au taux de fragmentation de l'ADN spermatique.

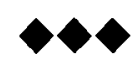

Evaluation du risque chromosomique par hybridation in situ fluorescente (FISH) des spermatozoïdes des hommes porteurs de translocations équilibrées: intérêt pour la prise en charge en AMP. Etude à propos de deux cas

\author{
M. BERGERE ${ }^{1}$, F. VIALARD ${ }^{1}$, D. MOLINA- \\ GOMES ${ }^{1}$, G. SOUDRE ${ }^{2}$, A. DE LANETE ${ }^{1}$, \\ J. SELVA ${ }^{1}$. \\ 1 Service d'Histologie-Embryologie-Cytogénétique \\ Centre Hospitalier Poissy Saint Germain en Laye \\ ${ }^{2}$ Centre d'AMP CMC Parly II Le Chesnay
}

Introduction :

Les différents remaniements chromosomiques équilibrés constitutionnels ont un retentissement variable sur la méiose, et la proportion de gamètes chromosomiquement équilibrés obtenus. Les données disponibles quant aux risques pour la descendance sont obtenues directement à partir des couples fertiles ( Réciconseil), ces données ne sont pas nécessairement les mêmes pour les couples infertiles. L'étude des spermatozoïdes par FISH préalable à une AMP permet de préciser la proportion de gamètes chromosomiquement normaux ou équilibrés, et, en fonction de ce pourcentage de cerner, avec le couple, les opportunités respectives du DPI et des différents types de diagnostics chromosomiques anténatals.

\section{Matériel et Méthodes :}

L'analyse du sperme de $\mathrm{Mr} \mathrm{B}$, porteur d'une translocation robertsonienne $\mathrm{t}(13 ; 14)(\mathrm{q} 10 ; \mathrm{q} 10)$, a été réalisée avec une sonde télomerique du $14 q$ ( marquée directement à la cyanine 3) et une sonde d'une séquence unique du 13q (LSI 13, marquée directement à la fluoresceine).

L'analyse du sperme de Mr E, portcur d'une translocation réciproque $\mathrm{t}(8 ; 14)(\mathrm{p} 21 ; \mathrm{q} 23)$, a été réalisée avec une sonde télomerique du 8p (marquée à la fluoresceine) et une sonde télomerique du 14q (marquée à la cyanine 3).

\section{Résultats :}

Mr B : 62 spermatozoïdes ont été analysés, $57 \%(\mathrm{n}=35)$ étaient normaux ou équilibrés, parmi les $40 \%$ de spermatozoïdes déséquilibrés, $10 \%(\mathrm{n}=6)$ étaient porteur d'une disomie 13 et donc susceptibles de générer un embryon trisomique 13.

Mr E : 138 spermatozoïdes ont été analysés, 30\% $(n=41)$ étaient normaux ou équilibrés, $70 \%(\mathrm{n}=97)$ étaient déséquilibrés, la ségrégation $3 / 1$ étant la plus fréquente, $50 \%$ des cas $(n=66)$. Compte tenu de la petite taille des segments transloqués, les embryons conçus avec ces spermatozoïdes déséquilibrés pourraient avoir un développement prolongé.

\section{Conclusion :}

Pour le couple B le risque d'un fotus chromosomiquement déséquilibré viable était estimé à $10 \%$. Le couple s'est orienté d'emblée vers l'ICSI et le diagnostic anténatal. Pour le couple $E$, dont le risque était plus élevé, le diagnostic préimplantatoire a été suggéré, mais le couple a renoncé, et s'est dirigé vers l'AMP. Des études complémentaires sont en cours afin de juger de l'effet éventuel des différentes méthodes de préparation des spermatozoïdes sur le pourcentage de spermatozoïdes chromosomiquement normaux mis en fécondation, et d'adapter ainsi le traitement du sperme le jour de l'AMP.

Impact des traitements androgéniques sur la sexualité chez 26 hommes avec dysfonction érectile et taux abaissé de testostérone selon que la testostérone totale, ou exclusivement ses fractions libre ou biodisponible, étaient diminuées

\section{J. BUVAT, A. LEMAIRE., B. ALEXANDRE., J. RATAJCZYK}

Centre d'Etude et de Traitement de la Pathologie de I'Appareil Reproducteur et de la Psychosomatique, 3 Rue Carolus, Lille, France.

Introduction :

La responsabilité du déficit androgénique dans les dys- 
fonctions érectiles (DE) est encore discutée. En effet le traitement androgénique n'obtient que des résultats modestes chez les hommes avec DE et taux abaissé de testostérone plasmatique $(\mathrm{T})$. Il a été proposé que le taux des testostérones dites libre (TL) et biodisponible (TB $=\mathrm{T}$ non liée à la Sex Hormone Binding Globulin) constituent des critères d'androgénicité plus fiables que celui de la testostérone totale (TT), la plus couramment dosée, et devraient lui être préférés pour dépister les patients susceptibles de tirer bénéfice d'un traitement androgénique. Dans une première étude, rétrospective, et comportant une évaluation exclusivement clinique, ces dosages n'avaient pas eu d'avantage sur celui de la TT. Cette étude voulait vérifier nos résultats antérieurs de façon cette fois prospective, et en utilisant l'International Index of Erectile Function (IIEF) pour une évaluation plus objective.

\section{Matériel et Méthodes :}

Les patients étaient 26 hommes de 38 à 68 ans remplissant les 4 critères suivants : 1) DE depuis plus de 6 mois ; 2) pas de cause évidente autre qu'hormonale ; 3) dosages de TT, TL et TB par méthode radio-immunologique, à deux reprises ; 4) chaque fois au moins un des trois taux en-dessous des limites de la norme pour les hommes de 40 à 50 ans. Ces sujets ont reçu un traitement androgénique pendant trois mois : soit Enanthate de Testostérone, $250 \mathrm{mg}$ IM toutes les deux semaines $(n=11)$, soit Undécanoate de Testostéronc, $80 \mathrm{mg}$ per os matin et soir $(\mathrm{n}=7)$, soit Gonadotrophines Chorioniques, 5000 UI IM deux fois par semaine $(\mathrm{n}=8)$, dans ce dernier cas après qu'il ait été vérifié que la $\mathrm{T}$ était normalisée trois jours après une injection. L'IIEF fut administré avant et après trois mois de traitement. Les principaux critères utilisés furent le score total des 15 questions (IIEF 15), celui des 5 premières questions (IIEF 5), le Domaine Fonction Erectile (Q1-5 + 15 = IIEF 6) et le Domaine Désir (Q11 + Q12). Les effets sur la fonction érectile et le désir sexuel furent également évalués par l'interrogatoire, selon des critères quantitatifs.

\section{Résultats :}

TT était basse $(<3 \mathrm{ng} / \mathrm{ml})$ che $\% 15$ hommes, TL $(<16$ $\mathrm{pg} / \mathrm{ml})$ chez 19 , et $\mathrm{TB}(<0.75 \mathrm{ng} / \mathrm{ml})$ chez 19 . Les trois dosages étaient bas chez 11 (groupe 1), seules TB et / ou TL étaient basses chez 11 (groupe 2), enfin seule TT était basse chez 4 (groupe 3). Dans l'ensemble, l'évaluation clinique releva une amélioration franche des érections chez 12 hommes sur $26(46 \%)$ et une amélioration franche du désir sexuel chez 7 des 11 hommes chez qui il était initialement diminué $(63 \%)$. Cependant seuls 3 hommes sur 26 jugèrent leurs érections normalisées, et 2 sur 15 leur désir normalisé. Le score du Domaine Fonction Erectile augmenta de $\geq 6$ sur un total possible de $30 \mathrm{chez} 54 \%$ des patients, de $\geq 9$ chez $27 \%$ et de $\geq 12$ chez $11 \%$. Les scores moyens de l'IIEF $5(+5.3=+52 \%)$, de l'IIEF $6(+$
$6.15=+52 \%)$ et de l'IIEF $15(+11.5=+35 \%)$ augmentèrent de façon hautement significative, mais il n'y eut pas de modification du score moyen du Domaine Désir. L'augmentation des scores d'érection fut significative avec chacun des 3 traitements. L'amélioration de la fonction érectile fut très nettement supérieure dans le groupe 1 par rapport aux groupes 2 et 3 : amélioration clinique franche chez respectivement 10 hommes sur 11,2 sur 11 et 0 sur 4 , augmentation $\geq 6$ de l'IIEF 6 chez 8 sur 11,4 sur 11 et 2 sur $4, \geq 9$ che\% 5 sur 11,2 sur 11 et 0 sur 4 . De plus, parmi les 21 hommes de plus de 50 ans, les scores moyens des IIEF 5, 6 et 15 n'augmentèrent de façon significative que dans les groupes 1 et $1+3$, mais pas dans le groupe 2 .

\section{Conclusion :}

Dans cette étude ouverte, le traitement androgénique a amélioré de façon significative les scores moyens de l'IIEF et de son Domaine Fonction Erectile chez les hommes avec DE et TT abaissée de façon répétée, mais pas chez les hommes avec abaissement exclusif de TB et/ou TL mais taux normal de TT. Ceci ne plaide pas en faveur de la supériorité des dosages de TL et TB sur celui de TT pour dépister les DE d'origine réellement hormonale. Le score du Domaine Fonction Erectile s'avéra bien corrélé à l'évaluation clinique des érections. Par contre la discordance entre l'amélioration clinique du désir et l'absence d'augmentation des scores du Domaine Désir de l'IIEF fait douter de la validité de ce score pour évaluer le désir, au moins dans la version française de l'IIEF.

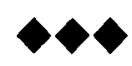

\section{La préparation du sperme par gradient de den- sité modifie-t-elle le taux et le type d'anomalies morphologiques?}

P. COHEN-BACRIE1, M. PLACHOT2, J. DE MOUZON3

1Laboratoire d'Eylau 55 rue saint Didier 75116 Paris. 2 Laboratoire de Fécondation in vitro $\mathrm{CHI}$ Jean Rostand 141 Grande Rue 92311 Sèvres. 3 Unité INSERM 569, 82 rue du général Leclerc 94276 Kremlin Bicêtre

\section{Introduction :}

Plusieurs méthodes de préparation du sperme ont été proposées en vue d'assistance médicale à la procréation : migration-sédimentation, swim-up, centrifugation sur gra- 
dient de densité, colonne d'albumine, filtration sur laine de verre, etc. Toutes montrent une amélioration du taux global d'atypie, variant suivant les techniques et les auteurs de 6 à 25\%. En revanche, peu d'articles montrent, sur un effectif suffisant, quels types d'anomalies sont diminués après préparation.

Le but de ce travail est d'évaluer les variations de chaque type d'anomalies avant et après centrifugation du sperme sur gradient de densité chez des patients explorés en vue d'une tentative d'assistance médicale à la procréation.

\section{Matériel et Méthodes :}

309 tests de migration survie ont été réalisés par gradient de Puresperm® ( 2 couches, $45 \%$ et $90 \%$ ) chez des patients qui présentaient une variation du spermocytogramme avant et après migration au moins égale à $10 \%$. Elle s'échelonnait avant migration entre $35 \%$ et $100 \%$. Dans 58 cas d'oligoasthénospermie sévère, l'incubation n'a duré que $2 \mathrm{H}$ au lieu des $17 \mathrm{H}$ habituelles.

L'analyse morphologique des spermatozoïdes est effectuée sur un frottis après fixation et coloration de Schorr. Une classification à entrées multiples est nécessaire pour tenir compte de l'existence de plusieurs anomalies sur le même spermatozoïde. La classification française de David répartit les anomalies en 13 groupes différents dont 7 anomalies de la tête, 2 anomalies de la pièce intermédiaire et 4 anomalies flagellaires. L'index d'anomalies multiples (IAM) est calculé de la façon suivante : IAM = nombre total d'anomalies ( associées + isolées ) divisé par le nombre de spermatozoïdes atypiques.

\section{Résultats :}

\begin{tabular}{|c|c|c|c|}
\hline & AVANT migration & APRES migration & $p+*$ \\
\hline 4typiques & 70.05 & 60.96 & 0,0001 \\
\hline Têtes allongées & 5.11 & 3.60 & $\mathrm{Ns}$ \\
\hline Têtes amincies & 9.08 & 8.09 & 0,02 \\
\hline microcéphales & 4.0 & 3.38 & Ns \\
\hline macrocéphales & 1.68 & 1.95 & Ns \\
\hline Têtes multiples & 0.99 & 0.87 & Ns \\
\hline Base anormale & 14.70 & 12.38 & 0.0004 \\
\hline acrosome malformé & 40.2 & 34.3 & 0.0004 \\
\hline restes cytoplasmiques & 1.67 & 0.48 & 0.0004 \\
\hline pièce intermédiaire amincie & 2.19 & 1.71 & 0.02 \\
\hline angulation & 16.80 & 12.64 & 0.0004 \\
\hline Nagelle absent & 0.136 & 0.39 & Ns \\
\hline nagelle ectopique & 6.65 & 4.19 & 0.0004 \\
\hline nagelle irrégulier & 5.04 & 2.39 & 0.0004 \\
\hline nagelle enroulé & 15.60 & 12.92 & 0.0004 \\
\hline Ragelles multiples & 1.15 & 0.70 & 0.0004 \\
\hline $\mathrm{AM}^{*}$ & 1.77 & 1.67 & 0.0004 \\
\hline \multicolumn{4}{|l|}{ Alypiques } \\
\hline Durée d'incubation $2 \mathrm{~h}$ & 79.9 & 71.44 & 0.001 \\
\hline Durée d'incubation $17 \mathrm{~h}$ & 67.39 & 57.70 & 0.001 \\
\hline
\end{tabular}

* : Index d'Anomalies Multiples

** : Comparaison de moyennes sur séries appariées test de Student

\section{Discussion :}

Ces résultats montrent que globalement le gradient de densité contre sélectionne les atypies spermatiques de façon significative en faisant passer la moyenne des atypies dans la zone limite de la normospermie. L'indice d'anomalies multiples est également diminué.

Tous les types d'anomalies sont significativement diminués après traitement sauf les flagelles absents et certaines anomalies de la tête. Comme on pouvait s'y attendre les anomalies du flagelle sont très diminuées après préparation ainsi que les anomalies de l'acrosome. Ces 2 paramètres sont essentiels en fécondation in vitro conventionnelle alors que seules les anomalies de l'acrosome pourront être limitantes en ICSI. Sur 7500 tests de migration survie chez des patients tout venant dans le cadre d'un bilan de stérilité il n'existe aucune différence significative dans la tératospermie avant et après préparation.

\section{Conclusion :}

Le gradient de densité diminue la tératospermie principalement par une diminution des anomalies du flagelle et de l'acrosome. Le taux de macrocéphales (chez lesquels on retrouve un taux élevé d'anomalies chromosomiques telles que la diploïdie) n'est cependant pas modifié dans cette étude.

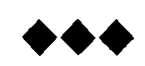

\section{Spermoculture et Assistance Médicale à la Procréation}

\author{
V. NAPOLY, M. DUMONT-HASSAN, A. M JUNCA, \\ P. COHEN-BACRIE
}

Laboratoire d'Eylau. Département de Bactériologie. 55 rue Saint Didier. 75116 Paris.

\section{Introduction}

L'exploration infectieuse réalisée chez un homme lors du bilan pré-AMP (Assistance Médicale à la Procréation) est nécessaire afin de définir au mieux sa part de responsabilité dans le bilan d'hypofertilité du couple et afin de prévenir les complications infectieuses lors de l'AMP en s'assurant de l'innocuité bactériologique du sperme.

Les résultats d'une étude rétrospective (sur 3 ans et 6 mois) sont présentés dans le but d'évaluer les risques d'échec d'une fécondation in vitro par contamination bacté- 
rienne des milieux de culture, en fonction de ou des spermocultures effectuées au préalable chez un patient.

\section{Matériel et Méthodes}

Durant la période concernée (années 1999, 2000, 2001 et 6 mois 2002) 24000 spermocultures ont été réalisées au laboratoire, quelque soit le contexte clinique (infection, hypofertilité, contrôle après traitement antibiotique, bilan pré-AMP...) et 12472 tentatives de fécondation ont été effectuées (5893 FIV et 6579 ICSI).

\section{Résultats :}

\section{Spermocultures}

2650 spermocultures $(11 \%)$ sont positives (identification et antibiogramme d'une ou plusieurs bactéries considérées comme pathogènes) avec une numération de germes comprise entre 200 et $200000 \mathrm{UFC} / \mathrm{ml}$. Les autres spermocultures $(89 \%)$ sont soit négatives soit positives avec présence d'une flore bactérienne polymorphe et une numération de germes comprise entre 1000 et $200000 \mathrm{UFC} / \mathrm{ml}$. La présence de Gardnerella vaginalis et/ou d'une flore anaérobie pluri-microbienne est classée dans cette dernière catégorie. La présence de levure a toujours été rattachée à une flore de contamination (mycose balano-préputiale. recueil effectué lors d'un rapport...) sans mise en évidence d'infection profonde à levure.

\section{Contaminations}

34 contaminations des milieux de culture ayant entraîné un arrêt de la fécondation ont été observées $(0.6 \%$ des tentatives).

Les germes retrouvés lors du contrôle bactériologique de ces milieux sont les suivants : Escherichia coli (34 cas), autres entérobactéries (Proteus mirabilis 2 cas. Klebsiella 1 cas) et entérocoque ( 1 cas). Il apparaît donc que seuls les bacilles à gram négatif de type entérobactérie et les entérocoques sont impliqués dans ces contaminations. Il n'a pas été isolé notamment de staphylocoque (doré ou non), de streptocoque autre que l'entérocoque (streptocoque du groupe B par excmple), de corynéhactérie (C. seminale étant pourtant souvent identifiée lor's des spermocultures), de Gardnerella vaginalis, d'Haemophilus (responsable

Tableau comparatif des 34 cas de contaminations FIV et des spermocultures effectuées chez le patient.

\begin{tabular}{|c|c|c|c|c|c|c|c|c|c|}
\hline & \multicolumn{2}{|c|}{$\begin{array}{l}\text { SPERMOCULTURE } \\
\text { Numération en UFC/ml }\end{array}$} & \multicolumn{2}{|c|}{$\begin{array}{c}\text { MILEUX FIV } \\
\text { Bactérie(s) isolée(s) }\end{array}$} & & \multicolumn{2}{|c|}{$\begin{array}{l}\text { SPERMOCULTURE } \\
\text { Numération en UFC/ml }\end{array}$} & \multicolumn{2}{|c|}{$\begin{array}{c}\text { MILIEUX FIV } \\
\text { Bactérie(s) isolée(s) }\end{array}$} \\
\hline & Date & Résultat & Date & Résultat & & Date & Résultat & Date & Résultat \\
\hline Cas 1 & $24 / 01 / 99$ & E.coli 1000 & $22 / 02 / 99$ & E.coli & Cas 14 & $\mathrm{NC}$ & $\mathrm{NC}$ & $31 / 10 / 00$ & E.coli \\
\hline Cas 2 & $\begin{array}{l}21 / 11 / 98 \\
25 / 03 / 99\end{array}$ & $\begin{array}{l}\text { E.coli } 5000 \\
\text { E.coli } 1000 \\
\end{array}$ & $19 / 03 / 99$ & E.coli & Cas 15 & $\mathrm{NC}$ & $\mathrm{NC}$ & $07 / 02 / 01$ & E.coli \\
\hline \multirow[t]{2}{*}{ Cas 3} & \multirow{2}{*}{$\begin{array}{l}13 / 02 / 99 \\
06 / 03 / 99 \\
\\
14 / 05 / 99 \\
10 / 06 / 99 \\
10 / 07 / 99 \\
\end{array}$} & \multirow{2}{*}{$\begin{array}{l}\text { Négative } \\
\text { E.coli } 1000 \\
+P m 1000 \\
\text { E.coli } 400 \\
\text { E.coli } 400 \\
\text { E.coli } 100 \\
\end{array}$} & \multirow[t]{2}{*}{$07 / 04 / 99$} & \multirow[t]{2}{*}{ Eccoli $+\mathrm{Pm}$} & Cas 16 & $07 / 06 / 01$ & $\begin{array}{l}\text { E.coli } 1000 \\
+ \text { Ko } 5000 \\
+ \text { EC } 3000 \\
\end{array}$ & $12 / 02 / 01$ & $\mathrm{E}$ coli $+\mathrm{Ko}$ \\
\hline & & & & & Cas 17 & $\mathrm{NC}$ & $N C$ & $19 / 03 / 01$ & E.coli \\
\hline \multirow[t]{2}{*}{ Cas 4} & \multirow{2}{*}{$\begin{array}{l}18 / 03 / 99 \\
21 / 06 / 99 \\
25 / 08 / 99 \\
\end{array}$} & \multirow{2}{*}{$\begin{array}{l}\text { Négative } \\
\text { Négative } \\
\text { Négative } \\
\end{array}$} & \multirow[t]{2}{*}{$23 / 04 / 99$} & \multirow[t]{2}{*}{ E.coli } & Cas 18 & $04 / 03 / 01$ & E.coli 1000 & $11 / 04 / 01$ & E.coli \\
\hline & & & & & Cas 19 & $\overline{\mathrm{NC}}$ & $\mathrm{NC}$ & $18 / 05 / 01$ & E.coli \\
\hline Cas 5 & $02 / 06 / 99$ & Négative & $28 / 06 / 99$ & E.coli & Cas 20 & $\mathrm{NC}$ & $\mathrm{NC}$ & $21 / 05 / 01$ & E.coli \\
\hline Cas 6 & NC & $\mathrm{NC}$ & $28 / 06 / 99$ & E.coli & Cas 21 & $23 / 01 / 99$ & Négative & $30 / 05 / 01$ & E.coli \\
\hline \multirow[t]{8}{*}{ Cas 7} & \multirow[t]{2}{*}{$21 / 08 / 99$} & \multirow{8}{*}{$\begin{array}{l}\text { E.coli } 10000 \\
+ \text { Pm } 10000 \\
+ \text { En } 5000 \\
\text { E.coli } 4000 \\
+ \text { Pm } 6000 \\
\text { E.coli } 10000 \\
\text { Pm } 10000 \\
\text { E.coli } 10000 \\
+ \text { Pm } 10000 \\
+ \text { En } 10000 \\
\text { Pm } 15000\end{array}$} & \multirow[t]{8}{*}{$07 / 10 / 99$} & \multirow[t]{8}{*}{ E.coli +Pm } & Cas 22 & $07 / 06 / 01$ & E.coli 20000 & $23 / 06 / 01$ & E.coli \\
\hline & & & & & Cas 23 & $11 / 06 / 01$ & Ecoli 10000 & $27 / 10 / 01$ & E.coli \\
\hline & $27 / 11 / 99$ & & & & Cas 24 & $26 / 10 / 01$ & E.coli 1000 & $15 / 11 / 01$ & E.coli \\
\hline & $04 / 02 / 00$ & & & & Cas 25 & $26 / 11 / 01$ & \begin{tabular}{|l|} 
Négative \\
\end{tabular} & $20 / 12 / 01$ & E.coli \\
\hline & $\begin{array}{l}19 / 09 / 00 \\
24 / 02 / 01\end{array}$ & & & & Cas 26 & $\begin{array}{l}12 / 10 / 01 \\
26 / 12 / 01 \\
\end{array}$ & $\begin{array}{l}\text { E.coli } 50000 \\
\text { E.coli } 5000 \\
\end{array}$ & $25 / 01 / 02$ & E.coli \\
\hline & & & & & Cas 27 & $18 / 07 / 01$ & Négative & $23 / 01 / 02$ & E.coli \\
\hline & \multirow[t]{2}{*}{$18 / 08 / 0 \mathrm{I}$} & & & & Cas 28 & $\mathrm{NC}$ & $\mathrm{NC}$ & $18 / 02 / 02$ & E.coli \\
\hline & & & & & Cas 29 & \begin{tabular}{|l|}
$13 / 11 / 01$ \\
$15 / 04 / 02$ \\
\end{tabular} & \begin{tabular}{|l|}
$\mathrm{Pm} 10000$ \\
Négative \\
\end{tabular} & $20 / 02 / 102$ & E.coli \\
\hline Cas 8 & $05 / 09 / 99$ & E.coli 5000 & $06 / 11 / 99$ & E.coli & \multirow[t]{2}{*}{ Cas 30} & \multirow[t]{2}{*}{$15 / 02 / 02$} & \multirow{2}{*}{$\begin{array}{l}\text { E.coli } 2000 \\
+\operatorname{Pm} 20000 \\
+ \text { En } 5000 \\
\end{array}$} & \multirow[t]{2}{*}{$25 / 03 / 02$} & \multirow[t]{2}{*}{ E.coli } \\
\hline Cas 9 & \begin{tabular}{|l|}
$03 / 02 / 99$ \\
$21 / 07 / 00$ \\
\end{tabular} & $\begin{array}{l}\text { Négative } \\
\text { E.coli } 5000 \\
\end{array}$ & $20 / 11 / 99$ & E.coli & & & & & \\
\hline Cas 10 & \begin{tabular}{|l|}
$11 / 09 / 99$ \\
$19 / 09 / 00$ \\
\end{tabular} & \begin{tabular}{|l|} 
Négative \\
Négative \\
\end{tabular} & $04 / 05 / 00$ & E.coli & Cas 31 & $\overline{N C}$ & $\mathrm{NC}$ & $31 / 03 / 02$ & E.coli \\
\hline Cas 11 & $04 / 05 / 00$ & $\begin{array}{l}\text { E.coli } 11000 \\
+ \text { En } 40000 \\
\end{array}$ & $03 / 06 / 00$ & E.coli & Cas 32 & $06 / 05 / 02$ & Négative & $22 / 07 / 02$ & E.coli \\
\hline Cas 12 & \begin{tabular}{|l|}
$15 / 02 / 00$ \\
$10 / 07 / 00$ \\
$11 / 10 / 00$ \\
\end{tabular} & $\begin{array}{l}\text { Négative } \\
\text { E.coli 1000* } \\
\text { Négative } \\
\end{array}$ & $29 / 07 / 00$ & E.coli & Cas33 & $29 / 12 / 01$ & $\begin{array}{r}\text { E.coli } 2000 \\
+P m 2000 \\
+ \text { En } 2000 \\
\end{array}$ & $22 / 06 / 02$ & E.coli \\
\hline Cas 13 & $\begin{array}{l}11 / 01 / 01 \\
20 / 11 / 01 \\
\end{array}$ & \begin{tabular}{|l} 
E.coli 50000 \\
Négative \\
\end{tabular} & $10 / 10 / 00$ & Ecoli + En & Cas 34 & $01 / 01 / 06$ & Négative & $28 / 06 / 02$ & E.coli \\
\hline
\end{tabular}

NC : spermoculture non effectuée au laboratoire. Résultat non connu.

E.coli (Escherichia coli). Pm (Proteus mirabilis). Ko (Klebsiella oxytoca). En (Entérocoque).

Ec (Enterobacter cloacae). 
d'urétrite), de Pseudomonas aeruginosa (responsable de prostatite chronique) ou de levure. Les recherches de Chlamydia trachomatis (par PCR) et de mycoplasmes (ureaplasma et mycoplasma hominis) sont négatives. La quantification des germes évaluée lors de ces contaminations (nombreuses colonies) est sans corrélation avec la numération de germes établie lors de la spermoculture du fait de méthodes de culture différentes.

\section{Discussion :}

\section{Cas des FIV contaminées malgré une spermoculture} négative

Il peut s'agir d'une spermoculture effectuée plus de 2 mois avant la FIV : dans ce cas il n'est pas possible d'être certain de l'innocuité du sperme le jour de la FIV car le délai est trop long. Il peut s'agir d'une spermoculture de contrôle après un traitement antibiotique pour un premier recueil positif. Ce premier traitement est souvent prescrit pour une durée trop courte ( 8 à 15 jours) pour permettre une guérison réelle mais suffit à inhiber de manière provisoire la croissance des bactéries lors de la spermoculture de contrôle, surtout si celle-ci est effectuée trop rapidement après l'arrêt du traitement ( $<8$ jours). Un délai plus long est en effet nécessaire pour permettre de mettre en évidence l'absence de guérison en identifiant de nouveau la même bactérie et éviter de rendre un résultat négatif chez ces patients. Il faut noter un cas $\left(\mathrm{N}^{\circ} 34\right)$ pour lequel la culture du sperme un mois avant et le jour même de la FIV était négative alors que les puits d'insémination et le milieu de culture d'embryon contenant les ovocytes étaient contaminés par E.coli, faisant alors suspecter une contamination des ovocytes lors de la ponction.

\section{Cas des FIV contaminées malgré une spermoculture fai- blement positive (numération $<1000 \mathrm{UFC} / \mathrm{ML}$ )}

Cela souligne la difficulté d'établir une valeur seuil pour interpréter un résultat dans le cadre d'un bilan pré-AMP : une faible numération peut-être liée à un traitement récent, à une contamination de recueil non significative d'infection a priori et non prise en compte par le médecin traitant, ou à une réelle infection chronique mais qui parfois ne s'exprime que faiblement lors d'une spermoculture. Dans ce dernier cas il faut tenir compte d'un délai d'abstinence trop court ( $<3$ jours) qui peut abaisser la concentration bactérienne du recueil étudié. Le caractère mono ou plurimicrobien d'une culture est important pour interpréter la présence d'une bactérie pathogène. Il faut différencier une culture de $400 \mathrm{UFC} / \mathrm{ml}$ d'E.coli au sein d'une flore plurimicrobienne de $10^{5}$ germes (rcflet d'une probable contamination urétrale évitable par des conditions de recueil strictes mais à ne pas traiter) d'une culture mono-microbienne de $400 \mathrm{UFC} / \mathrm{ml}$ d'E.coli obtenue lors d'un premier recueil (concentration suspecte et éventuellement à contrô- ler avant d'envisager un traitement) ou obtenue après traitement (témoin de l'absence de guérison).

3. Cas des FIV contaminées malgré une spermoculture positive significative (numération $>1000 \mathrm{UFC} / \mathrm{ml}$ )

Il s'agit le plus souvent d'une FIV réalisée après traitement d'un patient connu pour infection mais sans contrôler l'efficacité de ce traitement juste avant la tentative : lors d'une infection chronique la prise en charge thérapeutique est complexe et un traitement simple ( 8 - 15 jours) ne permettra pas la guérison.

La principale difficulté au vue d'un premier résultat de spermoculture est de déterminer s'il s'agit d'un résultat positif non significatif (contamination ponctuelle ?) ou de la mise en évidence d'une infection réelle. Le contexte psychologique particulier, la possibilité ou non d'effectuer des spermocultures de contrôle (acceptation du patient ?), le manque de temps éventuel (FIV programmée, facteurs liés à la conjointc...) sont autant de paramètres illustrant cette difficulté.

Un recueil de sperme effectué en cours de traitement peutêtre utile pour obtenir, de manière transitoire, une culture négative et permettre d'utiliser le sperme en AMP, sans attendre une (hypothétique) guérison dans le cas de ces infections chroniques.

Le choix des antibiotiques est également déterminant puisque certaines molécules (Fluoroquinolones, Bactrim ${ }^{R}$ ) sont plus à même d'être efficaces (pénétration prostatique notamment). Interviennent alors les problèmes de contreindications liées au patient, de sensibilité in vitro... Ainsi une infection à entéroque (naturellement résistant) ou à Escherichia coli ayant acquis une multi-résistance sera de mauvais pronostic quant à une guérison possible.

\section{Cas des FIV contaminées avec plusieurs germes}

Existence d'une bonne corrélation entre le ou les bactéries identifiées lors de la spermoculture et celles isolées lors de la culture des milieux de FIV. Si la plupart des contaminations sont mono-microbiennes, il a pu être cultivé jusqu'à trois espèces bactériennes dans le sperme et deux lors de la FIV. Un premier résultat de spermoculture avec deux ou trois germes pathogènes identifiés sans autre bactérie de contamination mérite d'être contrôlé avant tout traitement (éventuellement inutile car les infections pluri-microbiennes sont tout de même rares) ou avant toute FIV (car le risque de contamination est réel).

Il n'apparaît pas de contamination de FIV par la flore polymorphe commensale de l'urètre pourtant souvent présente dans le sperme, ce qui souligne le risque moindre que représentent ces prélèvements, à condition d'être certain de l'absence d'entérobactérie. 


\section{Conclusion :}

Le faible nombre de tentatives de fécondation interrompues du fait d'une contamination bactérienne durant des trois années confirme l'efficacité des mesures préventives prises pour s'assurer de l'innocuité des recueils, qu'il s'agisse des conditions strictes de prélèvement, de l'interprétation des résultats de spermoculture ou de la préparation des spermatozoïdes au laboratoire en vue de la fécondation in vitro.

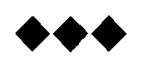

Evaluation clinique d'une nouvelle méthode d'enregistrement des érections nocturnes : intérêt du système NEVA

\section{A propos d'une série consécutive de 26 patients}

\author{
F. COLOMB, D. CHEVALLIER, G. GARCIA, \\ J. TOUBOL, J. AMIEL \\ Fédération Urologie Néphrologie (Pr Amiel), \\ $\mathrm{CHU}$ Nice
}

\section{Introduction :}

Dans notre expérience, l'enregistrement des érections nocturnes a largement sa place dans l'arbre décisionnel des patients consultant pour dysfonction érectile.

Le système NEVA est un nouvel outil pour expertiser les érections nocturnes avec des avantages par rapport aux rigidimètres existant sur le marché en terme de performance et d'ergonomie (faible encombrement).

Objectif : Tester la faisabilité , la valeur diagnostique du NEVA.

\section{Matériel et Méthodes :}

De Septembre 2000 à Mai 2001, 26 patients ont été explorés consécutivement à l'hôpital pour dysfonction érectile. Ils ont tous bénéficié d'un bilan d'évaluation standardisé (incluant autoquestionnaire IIEF) .

A l'issue de ce bilan les patients ont été répartis en 3 groupes : psycho-fonctionnalité dominante, organicité dominante et origine mixte. Secondairement, tous ces patients ont été hospitalisés 2 nuits pour un enregistrement des érections nocturnes avec le système NEVA.

Les courbes ont été relues à la lumière de la classification initiale afin d'établir une relation de cohérence entre les 2.

Résultats :

Les 26 patients ont été explorés et donc 52 enregistrements ont été réalisés.

La moyenne d'âge était de 54 ans (extrêmes 33-76). La durée moyenne des érections était de 19 minutes ; le nombre moyen d'érection de 3,4 par patient. Tous les patients ont accepté ce mode d'exploration et tous ont affirmé qu'il n'avait pas gêné leur sommeil. La sensibilité du NEVA pour reconnaître l'origine organique ou fonctionnelle de la dysfonction érectile était de $72 \%$ et la spécificité de $88 \%$, la valeur prédictive négative de $93 \%$.

Le NEVA a reconnu une insuffisance artérielle en précisant l'index de sévérité dans $7,5 \%$ des cas.

$\begin{array}{lll}\text { Troubles érectiles } & \begin{array}{l}\text { Classification } \\ \text { avant NEVA }\end{array} & \begin{array}{l}\text { Classification } \\ \text { après NEVA }\end{array} \\ \text { fonctionnels } & 14 & 13 \\ \text { organiques } & 6 & 10 \\ \text { mixtes } & 2 & 2 \\ \text { normaux } & 4 & 1 \\ \text { Conclusion : } & & \end{array}$

Le système NEVA paraît être un outil valide pour expertiser les érections nocturnes en réalisant 2 examens consécutifs ; les limites sont celles attribuées classiquement à la valeur de l'enregistrement nocturne et aux artéfacts (électrodes décollées) faciles à dépister.

Il permet une orientation diagnostique et thérapeutique et il est, grâce à son faible encombrement, parfaitement accepté par les patients.

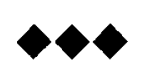

\section{Diagnostic d'une anéjaculation. A propos de 72 patients}

\author{
D. DELAVIERRE, M.H. HAPI, B. NSABIMBONA
}

Senvice Urologie-Andrologie, CHR d'Orléans

\section{Introduction :}

L'anéjaculation est définie par l'absence d'éjaculation par le méat urétral malgré une stimulation sexuelle appropriée et prolongée. La prévalence de l'anorgasmie (une des variétés d'anéjaculation) est respectivement évaluée dans les enquêtes ACSF (France 1993) et NHSLS (USA 1999) à 14 et $8 \%$. 


\section{Matériel et Méthodes :}

Du 1er/01/97 au 15/07/2002, 72 patients âgés de 17 à 72 ans (moyenne 43 ans ; $65 \%<50$ ans) ont consulté en raison d'une anéjaculation. 69 souffraient d'une altération de leur vie sexuelle ( 13 d'entre eux souhaitaient un enfant), 3 recherchaient uniquement des informations.

\section{Résultats :}

47 patients $(65 \%)$ ne présentaient pas d'orgasme (anorgasmie), 25 (35\%) pouvaient atteindre l'orgasme (éjaculation rétrograde 9 ou sèche 7 selon la présence ou non de sperme dans les urines après l'orgasme, anéjaculation indéterminée 7 ,éjaculation asthénique 2).

$40 \%$ des patients présentaient une dysfonction érectile, 19 $\%$ une altération du désir sexuel, $18 \%$ des troubles mictionnels.

$65 \%$ des patients ont bénéficié d'explorations complémentaires ( 45 bilans biologiques ayant permis de dépister 6 hypogonadismes, 12 explorations neurophysiologiques pelvipérinéales, 8 autres examens).

Les principaux facteurs de risque étaient psychologiques (36 patients, $50 \%$ ) (surtout contexte anxiodépressif, stress, difficultés conjugales, traumatisme psychologique après maladie ou intervention urogénitale, désir d'enfant mal assumé ou rejeté), neurologiques (34 patients, $47 \%$ ) (surtout neuropathies périphériques après chirurgie pelvienne ou rétropéritonéale 12 patients ou diabétiques 9 patients) et médicamenteux (16 patients $22 \%$ ) (surtout inhibiteurs de la recapture de la sérotonine 10 patients). 25 patients ( 35 $\%$ ) présentaient plusieurs facteurs de risque avec une prédominance psychologique chez 14 d'entre eux. Parmi les anorgasmies, $70 \%$ avaient des facteurs de risque psychologiques, $36 \%$ neurologiques et $34 \%$ médicamenteux contre respectivement $12 \%, 68 \%$ et $0 \%$ parmi les autres anéjaculations.

\section{Conclusion :}

1) Le diagnostic d'une anéjaculation nécessite dans un $1^{\text {er }}$ temps d'en préciser la variété.

2) Les facteurs de risque sont le plus souvent psychologiques, neurologiques et médicamenteux lors d'anorgasmie, le plus souvent neurologiques lors des autres anéjaculations.

3) Le diagnostic étiologique repose surtout sur l'interrogatoire.
Prévalence de la dysfonction érectile en France

\author{
D. DELAVIERRE
}

Service Urologie-Andrologie, CHR d'Orléans

\section{Enquête ACSF SPIRA/BEJIN (Analyse des Compor- tements Sexuels en France) (1993)}

Cette enquête téléphonique, réalisée de septembre 1991 à février 1992 en raison de l'épidémie de VIH, étudiait les comportements sexuels des français et comportait 7 questions sur les dysfonctions sexuelles. 1308 hommes âgés de 18 à 69 ans et ayant déjà eu au moins un rapport sexuel ont été interrogés. L'analyse des réponses, réalisée par Béjin, a fait apparaître que $28 \%$ de ces hommes avaient répondu «assez rarement» et $19 \%$ « souvent ou parfois » à la question « vous n'avez pas du tout d'érection ». Cette prévalence augmentait avec l'âge.

Les fréquences des autres dysfonctions, en ne considérant également que les réponses « souvent ou parfois », étaient les suivantes : éjaculation trop rapide $58 \%$, éjaculation tardive ou absente $27 \%$, absence ou insuffisance de désir sexuel $19 \%$, absence d'orgasme $14 \%$. Malgré les fréquences élevées de ces différentes dysfonctions $88 \%$ des hommes se sont déclarés (très ou assez) satisfaits de leur vie sexuelle.

\begin{tabular}{lcccccc} 
Age & $18-24$ & $25-34$ & $35-44$ & $45-54$ & $55-69$ & $\mathbf{1 8 - 6 9}$ ans \\
$\begin{array}{l}\text { Dysfonction } \\
\begin{array}{l}\text { érectile } \\
\text { "Souvent ou } \\
\text { parfois» }\end{array}\end{array}$ & $12 \%$ & $14 \%$ & $19 \%$ & $24 \%$ & $28 \%$ & $19 \%$ \\
\hline
\end{tabular}

Bejin A. - L'orgasme de l'homme adulte. Quelques résultats des études quantitatives récentes sur le comportement sexuel, notamment en France - Andrologie, 1997, 7(3): 336-342

Spira A, Bajos $N$ et le groupe ACSF - Les comportements sexuels en France - La documentation française, Paris, 1993.

\section{Enquête SOFRES/UPJOHN (1994)}

L'enquête de la SOFRES pour le Laboratoire UPJOHN a été réalisée en octobre 1994, selon la méthode des quotas, à l'aide d'un questionnaire renseigné par le patient luimême, auprès de 1000 hommes de 18 ans ou plus représentatifs de la population française. $45 \%$ reconnaissaient des problèmes d'érection dont $11 \%$ au moins une fois sur 2 avec une corrélation avec l'âge ( $4 \%$ entre 30 et 39 ans $25 \%$ après 65 ans). 
Age $\begin{array}{llllll}18-29 & 30-39 & 40-49 & 50-59 & \geq 60 & \geq 18\end{array}$

Dysfonction

érectile au

moins 1 fois

sur 2

Etude SOFRES sur l'impuissance masculine réalisée du 7 au 25 octobre 1994 pour les laboratoires Pharmacia Upjohn (échantillon de 1000 hommes $>18$ ans représentatifs de la population nationale).

\section{Enquête LERICHE/JAUDINOT (Patients à risque suivis en médecine générale) (2000)}

D’octobre 1999 en février 2000, 1845 médecins généralistes ont proposé à 7469 patients d'âge moyen 58,9 ans diabétiques $(31,8 \%)$, hypertendus $(52,3 \%)$ ou les $2(15,9 \%)$ des questionnaires sur leur fonction érectile : perception par le patient de sa fonction érectile et IIEF 5.

$60,9 \%$ des hypertendus, $67,4 \%$ des diabétiques et $77,9 \%$ des hypertendus diabétiques ont répondu «oui » à la question "Pensez vous souffrir d'un trouble de l'érection?» (scores IIEF respectifs 12 - 11,5 et 10,5). La prévalence augmentait avec l'âge et l'ancienneté du facteur de risque. $79,5 \%$ ont considéré ce trouble (assez ou très) gênant. 65 $\%$ des patients déclarant souffrir de troubles de l'érection n'étaient pas traités (dont $68,8 \%$ souhaitaient une prise en charge).

Leriche A, Jaudinot EO, Solesse de Gendre A.- Enquête sur la prévalence des troubles de l'érection et leur prise en charge chez des patients à risque suivis en consultation de médecine générale - Communication au $94^{\grave{e ̀ m e}}$ Congrès de l'Association française d'urologie, Paris, 15-19 Novembre 2000, Résumé 03.

\subsection{Enquête GIULIANO/SOFRES (2002)}

De mars à avril 2001 une enquête téléphonique a été réalisée par la SOFRFS sur un échantillon d'hommes représentatifs sur le plan socio-démographique. Les taux de refus de participation ( $85 \%$ ) et d'abandon en cours d'interrogatoire (28\%) ont été élevés. Au total 1004 hommes de plus de 40 ans ont répondu à une question générale ( Vous même, diriez vous que vous êtes sujet à des problèmes d'érection? »), à l'IIEF 5 et à des questions sur la répercussion de la dysfonction érectile éventuelle sur la qualité de vie.

$25 \%$ des hommes ont répondu «oui » à la question générale et $31,6 \%$ présentaient un score IIEF $5 \leq 20$ (dysfonction peu sévère $20 \%$ - moyenne $7,5 \%$ - sévère $4,1 \%$ ). La corrélation n'était pas parfaite entre les 2 méthodes d'évaluation : $31,8 \%$ des hommes ayant répondu oui à la question générale avaient en fait un score IIEF $5>20$ et $20 \%$ ayant répondu non un score IIEF $5 \leq 20$.

Avec l'âge la dysfonction érectile était non seulement plus fréquente (score IIEF $\leq 20: 11,7 \%$ entre 40 et 49 ans -
$66,8 \%$ au-delà de 70 ans) mais également plus sévère. 68 $\%$ des hommes ont déclaré qu'ils seraient insatisfaits à des degrés divers s'ils devaient passer le reste de leur vie avec ce problème érectile.

$\begin{array}{lccccc}\text { Age } & 40-49 & 50-59 & 60-69 & 70 \text { et plus } & \mathbf{4 0} \text { et plus } \\ \begin{array}{l}\text { Dysfonction } \\ \text { érectile(IIEF 5) }\end{array} & & & & & \\ \text { Peu sévère } & 9,6 \% & 18,8 \% & 24,8 \% & 33,2 \% & \mathbf{2 0} \% \\ \text { Moyenne } & 1,8 \% & 2,6 \% & 9,6 \% & 20,3 \% & \mathbf{7 , 5} \% \\ \text { Sévère } & 0,3 \% & 1,8 \% & 3,5 \% & 13,3 \% & \mathbf{4 , 1} \% \\ \text { \% Total } & 11,7 \% & 23,2 \% & 37,9 \% & \mathbf{6 6 , 8} \% & \mathbf{3 1 , 6} \%\end{array}$

Giuliano F., Chevret-Méasson M., Tsatsaris A., Reitz C., Murino M., Thonneau P. - Prévalence de l'insuffisance érectile en France : enquête épidémiologique menée auprès d'un échantillon représentatif de 1004 hommes.

\section{Phosphorylation des protéines, modification de la mobilité et remodelage des phospholipides membranaires associés à la capacitation des spermatozoïdes humains et murins}

\author{
B. DE VANTERY-ARRIGHI, A. SION, A. \\ DE AGOSTINI, F. URNER,D. CHARDONNENS, \\ H. LUCAS
}

Clinique de Stérilité et d'Endocrinologie Gynécologique, Département de Gynécologie et d'Obstétrique, Maternité, Hôpitaux Universitaires de Genève, 30, bd de la Cluse, 1211 Genève 14, Suisse.

\section{Introduction :}

Les processus de capacitation/réaction acrosomique des spermatozoïdes sont associés à des modifications ioniques (Ca2+ intracellulaire), des modifications dans la distribution et composition des lipides et des phospholipides membranaires, et des phosphorylations de certaines protéines permettant aux spermatozoïdes d'acquérir leur pouvoir fécondant.

\section{Matériel et Méthodes :}

Nous avons analysé au cours de la capacitation des spermatozoïdes humains et murins:

1) l'état de phosphorylation des protéines, protéines 
phosphorylées sur les résidus tyrosines (anticorps anti phosphotyrosine, clone $4 \mathrm{G} 10$ ), ainsi qu'une protéine spécifique décrite comme pouvant jouer un rôle dans les processus de capacitation/réaction acrosomique et/ou fusion avec l'ovocyte, la p42 MAP kinase (anticorps anti-ERK2 et anti-phospho-p44/p42MAPK) par western blotting.

2) la mobilité (\% de spermatozoïdes mobiles) et uniquement pour les spermatozoïdes humains, les caractéristiques du mouvement par la mesure des paramètres suivants : vitesse selon la trajectoire moyenne (VAP, $\mu \mathrm{m} / \mathrm{s}$ ), vitesse de progression linéaire (VSL, $\mu \mathrm{m} / \mathrm{s}$ ), vitesse curvilinéaire (VCL, $\mu \mathrm{m} / \mathrm{s}$ ), amplitude de déplacement latéral de la tête $(\mathrm{ALH}, \mu \mathrm{m})$, fréquence de croisement de trajectoire (BCF, battements/s), rectitude (STR=VSL/VAP), linéarité (LIN=VSL/VCL) et l'hyperactivation (HA) à l'aide d'un analyseur de mouvement Hamilton-Thorn;

3) identifié et quantifié le cholestérol (Chol) et les phospholipides membranaires, phosphatidyléthanolamine (PE), phosphatidylsérine (PS), phosphatidylinositol (PI), phosphatidylcholine (PC), et sphingomyéline (SM) par HPLC et « light-scattering detection»;

4) observé la présence et la répartition des phosphatidylsérines (PS) (exposition des PS à la surface de la membrane plasmique) par marquage à l'annexine-V-FITC et observation au microscope à fluorescence.

Les spermes humains de donneurs ont été préparés par gradient de centrifugation (PureSperm ${ }^{\circledR}$ ) et resuspension dans du milieu de culture T6 avec de l'albumine bovine (BSA) $(3 \mathrm{mg} / \mathrm{ml}$ ) (après préparation $=\mathrm{t} 0 \mathrm{~h}$ et $\mathrm{t} 3-5 \mathrm{~h}$ de capacitation). I.es spermatozoïdes de souris ont été prélevés dans la queue des épididymes de souris OF 1 et mis dans du milieu T6 avec BSA $(10 \mathrm{mg} / \mathrm{ml})$.

\section{Résultats :}

Dans nos conditions de préparation et de capacitation des spermatozoïdes humains et murins, nous avons confirmé qu'au cours de la capacitation un certain nombre de protéines (Mr 97, 82, 65, $55 \mathrm{kDa}$ ) sont phosphorylées sur les résidus tyrosines. Ces protéines phosphotyrosinylées sont localisées essentiellement dans la région flagellaire, mais aussi au niveau de la pièce intermédiaire et parfois dans la région de l'acrosome avec toutefois une variabilité selon les spermes utilisés. La présence et l'activation (phosphorylation) des $\mathrm{p} 42 \mathrm{MAPK}$ et $\mathrm{p} 44 \mathrm{MAPK}$ sont également variables d'un sperme à l'autre.

Parallèlement, nous avons analysé la mobilité des spermatozoïdes humains à l'aide d'un analyseur de mouvement (Hamilton-Thorn). Nous avons constaté une augmentation de la mobilité progressive et des paramètres VAP, VSL, VCL, ALH et du pourcentage d'HA au cours de la capacitation. L'analyse des lipides membranaires des spermatozoïdes humains et murins, a permis de quantifier les lipides membranaires, le Chol et les phospholipides PE, PS, PI, $\mathrm{PC}$, et SM, présents avant et après capacitation. Chez la souris, nous montrons une prédominance de la classe des phospholipides PC et une faible représentation des phospholipides PI, comme décrit précédemment pour le sperme humain natif. En outre, pour les spermatozoïdes humains et murins, les PS sont externalisés au cours de la capacitation. En effet, le pourcentage, variable selon les spermes utilisés, de spermatozoïdes marqués à l'annexineV-FITC augmente au cours de la capacitation. Les PS sont localisés soit au niveau de la tête, du col, de la pièce intermédiaire et/ou du flagelle des spermatozoïdes.

\section{Effets de la décondensation de la chromatine et des cassures de l'ADN des spermatozoïdes humains sur les taux de fécondation en ICSI}

\section{DE VANTERY-ARRIGHI, H. LUCAS, D. CHAR- DONNENS, A. CAMPANA, P.G. BIANCHI}

Clinique de Stérilité et d'Endocrinologie Gynécologique, Département de Gynécologie et d'Obstétrique, Maternité, Hôpitaux Universitaires de Genève, 30, bd de la Cluse, 1211 Genève 14, Suisse.

\section{Introduction :}

La qualité du sperme humain est généralement évaluée dans les laboratoires d'Andrologie par les paramètres classiques du spermogramme (volume, morphologie, concentration, mobilité) définis par l'OMS (1999). Cependant, la qualité nucléaire (état de condensation de la chromatine et fragmentation/cassures de l'ADN) des spermatozoïdes pourrait-être un indicateur important du pouvoir fécondant des spermatozoïdes.

Les objectifs de cette étude étaient d'évaluer : 1) les effets d'une préparation du sperme natif par PureSperm® sur la sélection des spermatozoïdes à morphologie normale, 2) l'incidence des anomalies de l'ADN (condensation, fragmentation) après une préparation du sperme natif au PureSperm $®$ et de corréler : 3 ) la présence dans les spermatozoïdes de chromatine décondensée et/ou de cassures de l'ADN avec les paramètres classiques du spermogramme (morphologie, concentration et mobilité) et de corréler : 4) la présence dans les spermatozoïdes de chromatine décondensée 
ou de cassures de l'ADN avec les taux de fécondation en ICSI.

\section{Matériel et Méthodes :}

\section{Patients :}

Les spermes éjaculés/natifs, préparés par PureSperm®, sont obtenus à partir de 34 patients consultant la Clinique de Stćrilité des Hôpitaux Universitaires de Genève, Suisse, pour un traitement par ICSI.

\section{Méthodes:}

Les anomalies de la condensation de la chromatine et les cassures de l'ADN des spermatozoïdes sont évaluées, par respectivement, le marquage à la chromomycine A3 (CMA3) et par la technique de « in situ nick translation assay ».

\section{Résultats :}

Notre étude montre une corrélation inversée entre les paramètres classiques du sperme (concentration, mobilité et morphologie) et les anomalies nucléaire (chromatine décondensée et cassures de l'ADN). Par contre. nous n'avons pas trouvé de corrélation statistiquement valable entre les paramètres classiques du sperme ou les anomalies de la chromatine et les taux de fécondation en ICSI. Nos résultats confirment également que : 1) la préparation du sperme natif par PureSperm(B) permet d'enrichir la population de spermatozoïdes en formes morphologiquement normales et 2) les techniques de marquage au CMA3 et de « in situ nick translation » peuvent-être utilisées pour évaluer la qualité de la chromatine des spermatozoïdes.

\section{Conclusion :}

L'investigation de l'état de condensation de la chromatine et/ou de la présence de cassures de l'ADN contribuerait à améliorer la caractérisation du sperme. Malgré l'abscence de corrélation statistiquement significative entre l'intégrité nucléaire et le pouvoir fécondant des spermatozoïdes en ICSI, les taux de fécondation montrent une tendance à être : 1) plus faible pour les spermes avec un taux de fragmentation élevé et 2) plus élevé pour les spermes avec un taux de fragmentation faible. Une étude à plus grande échelle serait nécessaire pour conclure sur l'utilité d'analyser l'état de condensation de la chromatine et/ou les cassures de l'ADN dans l'estimation du pouvoir fécondant des spermatozoïdes.

\section{"FertiGenNet"}

\section{Le réseau Génétique et Infertilité}

\author{
A.-K. FAURE. \\ Laboratoire de Biologie de la Reproduction, $\mathrm{CHU}$ \\ Grenoble
}

Un nouveau réseau, le réseau "FertiGenNet", vient de naître dans le domaine de la reproduction. Son but est de réunir les cliniciens, biologistes et chercheurs intéressés par les aspects génétiques et épigénétiques de la reproduction. Afin de promouvoir ce réseau et de le faire vivre, nous avons créé un site internet disponible à l'adresse suivante :

\section{http://fertigennet.free.fr}

Le site comporte deux parties. La première, accessible à tous les internautes, présente le réseau "FertiGenNet", offre la possibilité de s'inscrire en ligne, et propose des informations et des liens concernant les aspects génétiques et épigénétiques de la reproduction. Il permet notamment de rester à jour concernant les pratiques dans ce domaine de l'infertilité (détection des délétions du chromosome $\mathrm{Y}$, anomalies cytogénétiques associées aux infertilités, ...).

La deuxième partie est un espace réservé, accessible uniquement aux professionnels qui se sont inscrits au réseau. Il s'agit d'un forum qui regroupe des informations sur chacun des membres du réseau "FertiGenNet" (coordonnées professionnelles, techniques maîtrisées, domaines de recherche et projets en cours). Ce forum permet aux membres du réseau de prendre rapidement les contacts adéquats dans les domaines de recherche qui les intéressent, que ce soit à l'échelle nationale ou internationale, et ainsi leur donne l'opportunité de mettre en place des collaborations scientifiques, des projets de recherche multicentriques ou d'échanger des renseignements divers.

Le réseau "FertiGenNet" pourra ainsi servir de base à la mise en place de collaborations entre chercheurs, biologistes et cliniciens sur le thème des aspects génétiques et épigénétiques de la reproduction et des infertilités, et les aidera à se préparer à répondre à des appels d'offre pour des projets nationaux ou internationaux, par exemple Européens (du type PCRD).
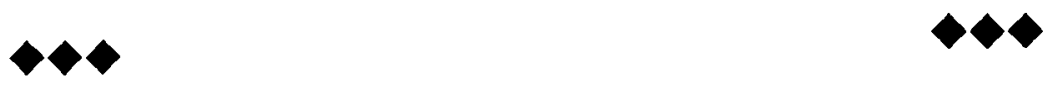


\section{Une forme de cryptorchidie d'origine génétique : le syndrome de persistance des canaux de Müller}

\section{BELEVILLE1, J. GONZALES1,2, S. IMBEAUD1,} N. DI CLEMENTE1, N. JOSSO1, J.Y. PICARD1

\section{$1 U 493$ INSERM, ENS, Montrouge et 2 Pitié-Salpêtrière}

\section{Introduction :}

Le syndrome de persistance des canaux de Müller (PMDS) est une forme rare de pseudohermaphrodisme masculin qui se manifeste par la présence de trompes de Fallope et d'un utérus chez un sujet 46,XY aux organes génitaux externes normaux.

Les circonstances de découverte sont chirurgicales, soit lors d'une intervention pour cryptorchidie, soit lors d'une cure de hernie inguinale qui est souvent le signe d'appel d'un PMDS.

\section{Matériel et Méthodes :}

L'étude génétique de 76 familles provenant de différents pays à travers le monde a permis de répondre en partie à l'étiopathogénie de ce syndrome. Trois situations doivent être distinguées.

\section{Résultats :}

1. L' hormone anti-Müllérienne (AMH) n'est pas détectable par le dosage ELISA dans le serum de ces patients. Cette glycoprotéine homodimérique de $140 \mathrm{kDa}$ fait partie de la famille du Transforming Growth Factor (TGFß). Elle subit un clivage protéolytique au niveau d'un site monobasique se trouvant dans le dernier tiers de la protéine donnant le domaine $\mathrm{C}$ terminal biologiquement actif de 25 $\mathrm{kDa}$ (109 acides aminés) et la partie $\mathrm{N}$ terminale inactive mais qui potentialise l'activité de la précédente. L'AMH codée par un gène de $3 \mathrm{Kbp}$ porté par le chromosome 19 (19p13.3) présente des mutations dans $47 \%$ (36/76) des familles PMDS.

2. Pour un deuxième groupe de patients, le PMDS est dû à une insensibilité à l'AMH, par anomalie de son récepteur de type II (AMHR-II). Le taux d'AMH sérique est normal ou élevé pour l'âge. Cette glycoprotéine de $85 \mathrm{kDa}$ dans sa forme mature glycosylée est codée par un gène localisé sur le chromosome 12 (12q13). Ce récepteur a un domaine transmembranaire unique et il possède un domaine extracellulaire sur lequel se lie l'AMH et un domaine intra-cellulaire portant l'activité sérine/thréonine kinase. Chez 38 $\%$ de ces familles (29/76), des anomalies ont été observées sur la totalité de ce récepteur.

3. Un troisième groupe de 11 familles est constitué de patients pour qui aucune anomalie génétique de l'AMH ou de son récepteur de type II n'a été observée. Leur taux d'AMH sérique est souvent intermédiaire. Certains de ces patients porteurs d'un PMDS présentent des malformations extra-génitales, urinaires, abdominales. La ou les malformations serait-elle/seraient-elles associécs à une anomalie génétique lors du clivage de l'AMH ou dans la cascade de transduction du signal de l'AMH en aval de son récepteur de type II ? Sont-ce des syndromes malformatifs sans lien direct avec les facteurs responsables de la régression müllérienne? Des études complémentaires portant sur une plus grande série de patients seront nécessaires pour identifier les anomalies responsables du PMDS pour ces malades du troisième groupe, les données cliniques constituant un point essentiel dans cette approche.

Un conseil génétique est possible à partir de cet ensemble de résultats. Dans les cas où les mutations responsables ont été identifiées, la transmission du PMDS est autosomique récessive, et il est possible d'établir le risque de PMDS chez un garçon à naître. Quelques cas de cancers testiculaires ont été par ailleurs rapportés chez des hommes pour lesquels la cryptorchidie a été trop longtemps négligée ou méconnue.

\section{Conclusion :}

Le PMISS constitue une forme particulière de cryptorchidie de transmission autosomique récessive. L'origine génétique a été prouvée dans des mutations des gènes AMH et AMHR-II et reste à déterminer dans quelques cas.

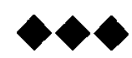

Recherche d'antigènes spermatiques pour la mise au point d'un vaccin contraceptif limitant la pullulation du campagnol terrestre Arvicola terrestris

\section{E. GRIGNARD, P. VERNET, J. DREVET}

Laboratoire Epididyme et Maturation des Gamètes, UMR 6547 CNRS-Université Blaise Pascal, 24 avenue des Landais, 63177 Aubière, France

\section{Introduction :}

Le campagnol terrestre Arvicola terrestris est un rongeur fouisseur, vivant dans les prairies des régions de moyenne montagne, où il provoque une très importante perte dans la 
production de fourrage. Depuis environ trente ans, le seul moyen de lutte contre la pullulation d'A. terrestris consiste en l'empoisonnement des animaux par un anticoagulant chimique disposé sur des appâts. En alternative à cet empoisonnement non spécifique qui occasionne des dégâts colatéraux dans les populations d'autres rongeurs et de leurs prédateurs, nous travaillons au développement d'un vaccin contraceptif ciblé uniquement sur Arvicola terrestris.

\section{Matériel et Méthodes :}

Nos études ont été effectuées sur des animaux capturés dans le Cantal entre Avril et Juillet 2002. Lors des douze séances de piégeage effectuées pendant cette période, le pourcentage de mâles varie entre $46 \%$ et $83 \%$, et la quantité de juvéniles n'a été supérieure à $50 \%$ que fin Avril et début Mai. Le poids moyen des mâles capturés est d'environ $90 \mathrm{~g}$.

Les spermatozoïdes de ces animaux ont une longueur d'environ $100 \mu \mathrm{m}$, avec une pièce intermédiaire de $20 \mu \mathrm{m}$ et une tête de $5 \mu \mathrm{m}$, coiffée d'un acrosome en forme de faucille.

\section{Résultats :}

La production d'anticorps polyclonaux dirigés contre des protéines spermatiques $\mathrm{d}$ ' $A$. terrestris nous a permis de mettre en évidence de nombreuses protéines spermatiques et des protéines présentes dans l'épididyme. Certaines de ces protéines, qui interviennent dans la fécondation, seraient susceptibles d'être des cibles d'un vaccin contraceptif. Ces anticorps reconnaissent principalement des protéines spermatiques (solubles et membranaires), de poids moléculaires $20-30 \mathrm{kDa}$, ainsi que des protéines de haut poids moléculaire. Une séparation des protéines spermatiques solubles selon leur point isoélectrique et leur poids moléculaire montre que les anticorps polyclonaux reconnaissent principalement des protéines acides et neutres dont les poids moléculaires sont compris entre $18 \mathrm{ct}$ $24 \mathrm{kDa}$, et $60-100 \mathrm{kDa}$, ou inférieurs à $16 \mathrm{kDa}$. Au niveau du spermatozoïde, une analyse immunocytologique montre une reconnaissance spécifique d'antigènes majoritairement au niveau de l'acrosome et de la gouttelette cytoplasmique. Par ailleurs, ces anticorps reconnaissent dans le testicule des cellules précurseurs des spermatozoïdes à des stades tardifs. Au niveau de l'épididyme, les spermatozoïdes sont reconnus par ces anticorps dans la tête, le corps et la queue. De plus des protéines présentes dans l'épithélium de la tête et la queue de l'épididyme sont reconnues.

\section{Hydrocéphalie récidivante en Tunisie}

\section{N. JABALLAH}

Policlinique CNSS, PB 418, Sousse, Tunisie

\section{Introduction :}

L'objectif de cette étude est l'investigation de la fréquence des hydrocéphalies parmi les nouveaux-né de parents consanguins en Tunisie et la comparaison de ces résultats avec ceux de la littérature.

\section{Matériel et Méthodes :}

Lne étude rétrospective de femmes accouchant d'enfants hydrocéphales a été faite. Les facteurs suivants ont été étudiés : anamnèse, déroulcment de la grossesse, examens de laboratoire et caryotype parental.

\section{Résultats :}

Les résultats de ces investigations ont été sans particularité. Les caryotypes parentaux sont normaux. Le seul facteur commun de ces cas est la consanguinité qui est fréquente en Tunisie et dans le monde arabe. L'étude de la littérature a démontré que les étiologies de l'hydrocéphalie récidivante sont congénitales dans $70.7 \%$ et acquise dans $29.3 \%$ des cas. Ces résultats sont respectivement 89.75 et $10.25 \%$ en Tunisie.

\section{Conclusion :}

L'hydrocéphalie récidivante est fréquente chez les parents consanguins. Un conseil génétique de ces couples est important, afin de réduire la fréquence de cette pathologie. L'étude de la localisation du gène de cette anomalie permettra la prévention de cette malformation.

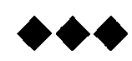

Etude du polymorphisme (CAG)n dans le gène du récepteur aux androgènes dans la population tunisienne et de son implication dans l'infertilité masucline

\section{HADJKCACEM 1; L. KESKES-AMMAR 1,2, H. HADJKCACEM1, A. ELGAIED-BOULILA1,} A. BAHLOUL2, H. AYADI1.

1: Laboratoire de Génétique Moléculaire Humaine de Sfax 2: Unité de santé "stérilité masculine" (US17) LGMH, Faculté de Médecine de Sfax, avenue Majida Boulila 3028, Sfax, Tunisie (lobna_hadjkacem@excite.com) 


\section{Introduction :}

Les androgènes sont des régulateurs importants de la spermatogenèse humaine. Ils agissent par l'intermédiaire d'un récepteur aux androgènes spécifique, codé par le gène $R A$, localisé sur le chromosome $X$ (Xq11-12). Le polymorphisme des répétitions $\mathrm{CAG}((\mathrm{CAG}) \mathrm{n})$ de l'exon 1 du gène $\mathrm{RA}$ est impliqué dans de nombreuses pathologies: maladie de Kennedy ( $C A G \geq 40)$, cancer de la prostate $(C A G<14)$ et infertilité masculine (variations modérées dans l'intervalle du polymorphisme normal (14-32)). Les objectifs de notre travail étaient l'étude du polymorphisme des répétitions CAG dans la population générale et l'étude d'association de ce polymorphisme avec l'infertilité masculine.

\section{Matériel et Méthodes :}

Le travail a porté sur un groupe de 129 hommes infertiles et un groupe témoin, composé de 98 hommes et 71 femmes fertiles, non apparentés. La région polymorphe (CAG)n du gène RA a été amplifiée par PCR. Les nombres de répétitions $C A G$ dans les produits $P C R$, ont été déterminés par comparaison avec des ADN de taille connue, après électrophorèse sur gel de séquence (polyacrylamide 6\%). L'analyse statistique des résultats était descriptive (moyenne \pm ES, histogramme et box-plot) et analytique (comparaison des moyennes par le test de student, comparaison des effectifs par le test de Kolmogorov-Smirnov (KS) et le test khi-carré).

\section{Résultats :}

Nous avons trouvé que le polymorphisme CAG du gène RA est significativement différent entre les femmes et les hommes fertiles. La moyenne des (CAG)n était plus élevée chez les femmes $(22.7 \pm 0.51$ vs $21.13 \pm 0.31, p=0.0096)$; le nombre d'allèle était également supérieur (20 vs 16), avec un intervalle variant de 13-32 pour les femmes et de 14 à 29 pour les hommes. Il n'existait pas de différences significatives entre les deux groupes des hommes fertiles et infertiles (moyennes: $21,13 \pm 0.31$ vs $20.8 \pm 0.27, p=$ 0,47 ; nombre d'allèles: 16 vs 17 et intervalle 14 à 29 vs 12-28). Les comparaisons par les tests $\mathrm{KS}$ et khi-carré n'ont pas montré de différences significatives $\left(\mathrm{D}_{\text {obs }}<\mathrm{D}_{\text {crit }} ; \mathrm{p}=0.32\right.$ ). La comparaison des sous-groupes des hommes infertiles (oligospermie, azoospermie et normospermie), a montré une différence significative entre les groupes oligospermique et azoospermique (moyenne: 21,6 $\pm 0,49$ vs $19,9 \pm 0,59$, respectivement; test de student: $p=$ 0,034; test khi-carré: $p=0,00079$ et test $\mathrm{KS}: \mathrm{D}_{\mathrm{obs}}>$ $\mathrm{D}_{\text {crit }}$ ).

\section{Conclusion :}

Il ressort de notre étude que le polymorphisme CAG dans l'exon 1 du gène RA des femmes est différent de celui des hommes, et que la comparaison entre les hom- mes fertiles et infertiles n'a pas montré d'association entre ce polymorphisme et l'infertilité masculine ainsi que les perturbations de la spermatogenèse. Dans la littérature, des résultats contradictoires ont été rapportés et de nombreuses controverses persistent concernant l'implication de l'augmentation du nombre de CAG du gène RA dans l'infertilité masculine. Il est suggéré que ces controverses sont dues à des différences dans la caractérisation des groupes d'études et à des différences des origines ethniques.

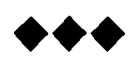

Utilisation des ARNm de la clusterine comme marqueur de la quantité d'ARNm d'origine sertolienne dans les biopsies testiculaires - Application à la quantification des ARNm de Stem Cell Factor dans les troubles de la spermatogenèse.

\author{
I. PLOTTON, P. SANCHEZ, M.H. PERRARD- \\ SAPORI, P. DURAND, H. LEJEUNE. \\ INSERM-INRA U 418, Hôpital Debrousse \& Départe- \\ ment de Médecine de la Reproduction, Hôpital \\ Edouard Herriot, Lyon, France.
}

\section{Introduction :}

La spermatogenèse est régulée par des facteurs paracrines produits par les cellules somatiques et les cellules germinales. La responsabilité éventuelle d'une altération de l'expression des facteurs paracrines dans les troubles de la spermatogenèse reste à démontrer. La quantification de l'expression des facteurs paracrines est rendue difficile par l'hétérogénéité cellulaire du testicule et les variations du nombre relatif des cellules somatiques et germinales dans les troubles de la spermatogenèse. Il a été montré que les ARNm de la clusterine sont localisés dans les cellules de Sertoli et ne sont pas modifiés par les gonadotrophines ni par la présence de cellules germinales en coculture. Nous avons cherché à évaluer si la quantité d'ARNm de la clusterine peut être un marqueur de la quantité des ARNm d'origine Sertolienne dans les biopsies testiculaires.

\section{Matériel et Méthodes :}

Trois conditions expérimentales induisant des variations du nombre relatif de cellules germinales et de cellules de Sertoli ont été étudiécs chez le rat : (1) le développement postnatal, (2) le traitement par l'acide methoxy-acétique (MAA), (3) le traitement par un agoniste du GnRH. La 
quantité d' $\Lambda \mathrm{RNm}$ de la clustérine mesurée par Northern Blot dans des cellules de Sertoli purifiées est stable à partir de l'âge de 20 jours et après traitement par le MAA ou le GnRHa. Nous avons alors mesuré par RT-PCR compétitive les ARNm de la clusterine et du Stem Cell Factor (SCF), un facteur paracrine produit par les cellules de Sertoli impliqué dans la prolifération et/ou la survie des cellules germinales, dans les ARN extraits de biopsies testiculaires dans les conditions expérimentales précédentes.

\section{Résultats :}

Le rapport ARNm du SCF / ARNm de la clusterine apparaît stable au cours du développement pubertaire, augmente lors de la repopulation des tubes séminifères après traitement par le MAA et diminue après GnRH agoniste. Ces résultats valident l'utilisation des $A R N m$ de la clusterine comme marqueur de la quantité d'ARNm d'origine sertolienne. Ces données nous conduisent à quantifier les ARNm de SCF par rapport aux l'ARNm de la clusterine, dans les biopsies testiculaires humaines. Chez les hommes avec une spermatogenèse normale $(n=4)$, la quantité d'ARNm de la clusterine est de $0,41 \pm 0,2110^{9}$ copies/?g ARN (intra and inter-assay CV: 12 and 78\%), la quantité d'ARNm du SCF est de $0,92 \pm 0,3110^{6}$ copies/?g ARN (intra and inter-assay CV: 18 and 67\%), et le rapport ARNm SCF/ARNm clustérine $=2,210^{-3}$. Les résultats préliminaires des mesures du rapport d'ARNm SCF/ARNm clusterine dans les biopsies testiculaires d'hommes infertiles indiquent que les taux d'ARNm SCF ne sont pas diminués dans les cas d'hypospermatogenèse $\left(3,210^{-3} ; n=2\right)$, d'aplasie des cellules germinales $\left(2,610^{-}\right.$ $3 ; n=2)$ ou de blocage de la spermatogenèse $\left(8,310^{-3}\right.$; $\mathrm{n}=1$ ), par rapport aux azoospermies obstructives à spermatogenèse normale $\left(2,610^{-3} ; n=2\right)$.

\section{Conclusion :}

Ces résultats préliminaires suggèrent qu'une diminution des taux d'ARNm de SCF n'est pas une caractéristique commune aux troubles de la spermatogenèse chez l'homme.

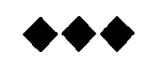

\section{Predisposition génétique aux cancers de testi- cule: les haplotypes du chromosome $Y$}

L. QUINTANA-MURCI1, M.E. WEALE2,3, M.G. THOMAS2, E. ERDEI4, N. BRADMAN2, J.H. SHANKS 5 , C. KRAUSZ ${ }^{1,6}$, K. MCELREAVEYI ${ }^{1}$
1Reproduction, Fertility and Populations, Institut Pasteur, Paris, France, 2 The Centre for Genetic Anthropology, Departments of Biology and Anthropology, University College London, University of London, London, UK., 3 Genostics Ltd, 28/30 Little Russell St, London WC1A 2HN, UK, 4Urology Unit, National Health Center, Budapest, Hungary, 5Department of Histopathology, Christie Hospital, Manchester, UK, 6 Andrology Unit, University of Florence, Florence, Italy

\section{Introduction :}

Le cancer du testicule est le cancer le plus fréquent chez les hommes jeunes dans beaucoup de pays. Par exemple au Danemark, le risque de développer un cancer du testicule est de $1 \%$ au cours de la vie. Plus de $97 \%$ des cancers du testicule ont pour origine les cellules germinales. Les gènes du chromosome $\mathrm{Y}$ sont nécessaires pour un bon développement et maintien des cellules germinales. Ceci suggére un implication du chromosome $\mathrm{Y}$ dans le cancer du testicule, un phénotype spécifiquement masculin. Ainsi le chromosome $Y$ posséde un locus (GBY) impliqué dans un type de cancer testiculaire, le gonadoblastome.

\section{Matériel et Méthodes :}

Afin de mettre en évidence une éventuelle corrélation entre le fond génétique du chromosome $\mathrm{Y}$ et la prédisposition au cancer du testicule, nous avons défini les haplotypes du chromosome $\mathrm{Y}$ chez un groupe d'hommes d'origine anglaise ayant développé un cancer testiculaire et nous avons comparé la distribution des fréquences de ces haplotypes entre ces patients et une population contrôle de la même origine géographique et ethnique.

\section{Résultats :}

Nos résultats suggèrent une absence d'effet majeur du fond génétique du chromosome $Y$ sur la prédisposition au cancer de testicule. Cependant, le rôle de ce chromosome dans la formation et la progression de ce type de cancer ne peux pas être totalement exclu.

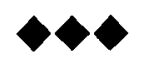

\section{Conséquences de la transmission des délé- tions du chromosome $Y$, implications pour le diagnostic pré-implantatoire}

\author{
L. QUINTANA-MURCI1, P.C. PATSALIS2, \\ C. SISMANI2, F. TALEB-BEKKOUCHE ${ }^{1}$,
}




\section{KRAUSZ1 1 K. MCELREAVEY1}

$1 /$ Immunogénétique Humaine, Institut Pasteur, Paris, France 2Cyprus Institute of Neurology and Genetics, Cyprus

\section{Introduction :}

Les délétions de certaines régions du chromosome $\mathrm{Y}$ sont responsables d'infertilité ou sub-fertilité chez l'homme. Cependant, des technologies récentes de fécondation in vitro permettent de pallier à ce problème en introduisant directement les spermatozoïdes (même présents à des concentrations faibles) du père dans l'ovocyte maternel. En principe, la seule conséquence de cette intervention est l'infertilité dans la descendance masculine car, par définition les déletions seront transmises du père au fils.

\section{Matériel et Méthodes :}

Nous avons étudié le chromosome Y d'un groupe de patients, sélectionnés à l'aveugle, avec un caryotype 45 $\mathrm{X} / 46 \mathrm{XY}$ et qui présentaient un phénotype Turner ou des ambiguiités sexuelles.

\section{Résultats :}

Nos résultats montrent qu'un tiers de ces patients présentent des délétions de la partie distale du chromosome $\mathrm{Y}$ communément observées chez les hommes infertiles. Ces résultats suggèrent que les traitements d'infertilité par fécondation assistée peuvent avoir des conséquences cliniques graves, autres qu'une infertilité attendue chez la descendance masculine, mais incluant potentiellement ambiguités sexuelles et syndrome de Turner. Pour mesurer le risque potentiel de ces interventions un diagnostic preimplantoire quand les hommes traités par ICSI présentent des délétions d'AZF, afin d'éviter le transfert d'embryons $45, X$. La plupart de ces embryons ne sont pas viables et sont associées aux avortements spontanés. En effet une augmentation des avortements spontanés a été notée après ICSI. En parallèle, des études de suivi chez des enfants concus par des techniques d'ICSI sont conseillées.

\section{Conclusion :}

Malgré le nombre faible des études concernant la transmission de microdélétions par des techniques de reproduction assistée, les résultats des karyotypes foetaux ICSI indiquent une augmentation significative des aneuplodies. Nos résultats suggèrent un méchanisme pouvant expliquer ces observations.

\section{Évidence génétique pour le syndrome de dysgénésie testiculaire}

\title{
Introduction :
}

L'augmentation du syndrome de dysgénésie testiculaire est liée au déclin global de la reproduction masculine, qui inclut une incidence élevée de cryptorchidisme, d'hypospadias, de baisse de la qualité du sperme et une incidence croissante des cancers du testicule. Nos données, issues de l'analyse de familles où co-ségrèguent des anomalies des organes génitaux externes et des dysgénésies gonadiques, suggèrent une étiologie génétique commune pour ces anomalies. Dans certaines familles, les différences phénotypiques peuvent être dues au fond génétique ou, comme suggérées par d'autres, causées par des facteurs environnementaux.

\section{Matériel et Méthodes :}

Dans cette étude, nous décrivons une famille avec des cas multiples de femmes 46,XY (avec une dysgénésie gonadique partielle ou pure), ainsi que des mâles 46,XY avec virilisation inachevée ayant pour résultat une hypospadia.

\section{Résultats :}

Une analyse complète du génome en utilisant des marqueurs microsatellites, nous a permis de localiser un gène impliqué dans le développement du sexe masculin dans une region de $6 \mathrm{cM}$ sur le chromosome 5 . Dans cet intervalle génétique, nous avons établi une carte physique d'environ $30 \mathrm{Mb}$ afin de rechercher des mutations dans les gènes candidats les plus probables de cette région.

\section{Le chromosome $\mathrm{Y}$ et la fertilité chez l'homme}

\author{
L. QLINTANA-MURCI ${ }^{1}$, E. RAJPERT-DE MEYTS2, \\ N. SKAKKEBAEK 2 , J. TOPPARI ${ }^{3}$, K. MCELREAVEY ${ }^{1}$ \\ 1 Reproduction, Fertilité et Populations, Institut Pasteur, \\ Paris, France, ${ }^{2}$ Growth and Reproduction, Rigshospitalet, \\ Copenhagen, Denmark, 3 Department of Pediatrics, Uni- \\ versity of Turku, Turku, Finland.
}




\section{Introduction :}

L'infertilité chez l'homme a été associée à une augmentation des anomalies du système urogénital mâle, incluant une cryptorchidie et une hypospadias ainsi qu'une augmentation de la fréquence des cancers du testicule. Ceci a conduit à l'utilisation du terme "syndrome de dysgénèse testiculaire". L'incidence de l'infertilité est estimée entre 10 et $17 \%$ de la population. Dans $50 \%$ de ces cas, c'est le partenairc masculin le responsable de l'infertilité du couple. Le chromosome $\mathrm{Y}$ porte un ou plusieurs facteurs nécessaires à la spermatogenèse, car des délétions du bras long du chromosome Y sont associées à des phénotypes d'infertilité masculine. Les raisons et les mécanismes responsables de la formation de ces délétions ne sont pas connus.

\section{Matériel et Méthodes :}

Nous avons réalisé trois études parallèles cas/témoin afin d'évaluer le rôle du fond génétique du chromosome $Y$ et le syndrome de dysgénésie testiculaire : (i) étude de prédisposition aux délétions du chromosome $Y$ causales des troubles d'infertilité et (ii) étude de prédisposition à la subfertilité dans la population danoise.

\section{Résultats :}

Nos résultats suggèrent une absence d'effet majeur du fond génétique du chromosome $Y$ sur la prédisposition aux microdélétions du chromosome $Y$. En revanche, nous avons identifié une lignée du chromosome $Y$ (haplogroupe 26) associée à une réduction significative de la concentration de sperme dans la population danoise. Nous sommes actuellement en train de rechercher si cette association retrouvée au Danemark est aussi observée dans des populations voisines, comme la Finlande et la Suède. En parallèle, nous sommes en train d'analyser les haplotypes du chromosome $\mathrm{Y}$ chez une population de mâles qui présentent une augmentation significative de la concentration de sperme.

Etude des causes génétiques associées aux anomalies du développement sexuel masculin par la technique "Comparative Génome Hybridisation"

\author{
K. MCELREAVEY1, L. QUINTANA-MURCI1, \\ V. PETITE1, B. LEVY2, H. OSTRER1 \\ 1Reproduction, Fertilité et Populations, Institut Pas- \\ teur, Paris, France; University School of Medicine, \\ New York, NY, USA
}

\section{Introduction :}

Des anomalies du développement sexuel masculin (dysgénésie gonadique, hypospadias et cryptorchidie) sont fréquentes, mais leur étiologie reste mal comprise. Nous avons présumé que certaines de ces anomalies peuvent résulter de remaniements chromosomiques (des gains ou des pertes) qui pourraient être détectés par l'hybridation génomique comparative ( $\mathrm{CGH}$ ).

\section{Matériel et Méthodes :}

D'abord, nous avons calibré la technique en analysant des cas de 46,XY av'ec dysgénésie gonadique avec des anomalies cytogénétiques connues en $9 \mathrm{p}$ et $1 \mathrm{lp}$. Ceci a été confirmé par l'hybridation in situ fluorescente.

\section{Résultats :}

Dans deux cas de 46,XX hermaphrodisme vrai, nous avons observé la présence d'une lignée de cellules avec un chromosome $\mathrm{Y}$, confirmant nos observations précédentes que de tels cas peuvent préscnter du mosaicisme ou du chimérisme. Dans un cas de 46,XX dysplasie campomélique, nous avons observé une duplication inversée de $17 \mathrm{p}$ distal de la région du gène $S O X 20$, non vue par la cytogénétique conventionnellc. D'autres gains et pertes chromosomiques ont été également détectés. Nous projetons d'élargir ces études à d'autres cas du dysfonctionnement de la reproduction masculine tels que l'infertilité masculine.

\section{Varicocélectomie et températures scrotales}

\author{
R. MIEUSSET, E. HUYGHE, F. PONTONNIER, \\ L.BUJAN. \\ Centre de Stérilité Masculine / Groupe de Recherche \\ en Fertilité Humaine, Hôpital La Grave, Toulouse, \\ France
}

\section{Introduction :}

Evaluer les températures scrotales chez les patients inféconds porteurs d'une varicocèle avant et après varicocèlectomic.

\section{Matériel et Méthodes :}

Chez 113 patients inféconds présentant une varicocèle clinique gauche ( $\mathrm{n}=101)$ ou bilatérale $(\mathrm{n}=12)$, les températures scrotales, rectales et ambiantes ont été mesurées avant, 
3 et 6 mois après cure chirurgicale de la varice. 116 hommes féconds sans varicocèle constituent le groupe témoin et servent à la définition de la limite supérieure de la température scrotale normal ( $\mathrm{d}$ et $\mathrm{g}: 35,1^{\circ} \mathrm{C}$ ).

\section{Résultats :}

Avant chirurgie. Les températures scrotales moyennes étaient significativement plus élevées chez les hommes inféconds avec varicocèle que chez les témoins. Les températures scrotales étaient normales chez 54 patients (47,8\% ; groupe normothermique) et anormalement élevées $\left(>35,1^{\circ} \mathrm{C}\right)$ chez 59 patients $(52,2 \%$; groupe hyperthermique ; unilatéral : $n=22$; bilatéral : $n=37$ ). Chez les patients inféconds avec varicocèle, les températures scrotales moyennes étaient significativement plus élevées dans le groupe hyperthermique que dans le groupe normothermique (d : $35,18 \pm 0,41$ vs $34,40 \pm 0,45^{\circ} \mathrm{C} ; \mathrm{g}: 35,50 \pm$ 0,31 vs $\left.34,60 \pm 0,44^{\circ} \mathrm{C}\right)$.

Après chirurgie. Les températures scrotales moyennes n'étaient plus différentes entre les groupes hyperthermique et normothermique à 3 mois ( $\mathrm{d}: 34,62 \pm 0,55$ vs $34,50 \pm$ $0,50^{\circ} \mathrm{C} ; \mathrm{g}: 34,60 \pm 0,50$ vs $34,46 \pm 0,52^{\circ} \mathrm{C}$ ) et à 6 mois (d : $34,72 \pm 0,42$ vs $34,44 \pm 0,45^{\circ} \mathrm{C} ; \mathrm{g}: 34,72 \pm 0,53$ vs $34,47 \pm 0,49^{\circ} \mathrm{C}$ ). Dans le groupe normothermique, une température scrotale anormale est observée chez $12 \%$ des patients à 3 mois et chez $4 \%$ supplémentaires à 6 mois. Dans le groupe hyperthermique, une température scrotale anormale est retrouvée $15 \%$ des patients à 3 mois et chez $12 \%$ supplémentaires à 6 mois.

\section{Conclusion :}

$50 \%$ des patients inféconds avec une varicocèle ont une température scrotale élevée. Après varicocèlectomie, les résultats sont satisfaisants en terme de population, les températures scrotales moyennes s'étant normalisées. Cependant, les valeurs individuelles sont beaucoup moins concluantes avec d'une part $16 \%$ des patients normothermiques devenant hyperthermiques après chirurgie, et d'autre part la persistance après chirurgie d'une hyperthermie préexistante chez $27 \%$ des patients.

\section{$\leftrightarrow \bullet$}

Pouvoir antibactérien du liquide séminal :

évaluation par la technique de quantification bactérienne

F. VIRECOULON 1, E. HUGUET 1, F. WALLET 2 , M.-C. PEERS 1, A. DEFOSSEZ 1, R. COURCOL 2, V. MITCHELL 1
${ }^{1}$ Laboratoire de Spermiologie/HistologieEmbryologie \& Biologie de la Reproduction et

2Laboratoire de Bactériologie-Hygiène, Hôpital Calmette, CHRU, 59037 Lille.

\section{Introduction :}

L'idée d'un pouvoir antibactérien (PAB) du liquide séminal (LS) est couramment admise. Pourtant, aucun consensus ne se dégage de la littérature très hétérogène : les études concernent de nombreuses espèces animales, portent sur des fractions différentes du LS, et utilisent des espèces différentes de bactéries. Pour l'espèce humaine, nous n'avons recensé que 3 études étudiant le PAB global, soit du sperme total [1], soit du LS [2, 3]. Ces 3 études montrent un $\mathrm{PAB}$ contre $S$. aureus variant de 5 à $78 \%$ ! De plus, si elles s'accordent pour retrouver $E$. coli globalement résistante, l'espèce a été montrée comme très fortement sensible à la séminalplasmine bovine et au liquide prostatique canin. Afin de faire le point, nous avons introduit une technique jamais employée pour l'évaluation du PAB du LS : la quantification bactérienne.

\section{Matériel et Méthodes :}

Trois souches bactériennes ( $S$. aureus, $S$. epidermidis et $E$. coli) isolées lors d'affections urogénitales sont testées visà-vis de 41 LS préalablement filtrés. Pour chacune, 3 concentrations bactériennes $\left(10^{3} / \mathrm{ml}, 5.10^{3} / \mathrm{ml}\right.$ et $\left.104 / \mathrm{ml}\right)$ sont incubées en parallèle soit avec le LS testé, soit avec de l'eau physiologique stérile. Chaque échantillon est ensuite étalé sur gélose (10 LS sur gélose BCP, 29 sur gélose au sang, et 2 sur les 2 géloses). Le dénombrement des colonies à 24 h permet d'établir le facteur d'inhibition (FI) : FI = nombre de colonies sur gélose témoin / nombre de colonies sur gélose patient, avec calcul d'un FI moyen $\left(\mathrm{FI}_{\mathrm{m}}\right)$ à partir des $\mathrm{FI}$ obtenus pour chaque concentration bactérienne. Ainsi, $\mathrm{FI}_{\mathrm{m}}=1$ : pas d'inhibition de croissance, $\mathrm{FI}_{\mathrm{m}}>1$ : inhibition de croissance, $\mathrm{F}_{\mathrm{m}}<1$ : stimulation de croissance.

\section{Résultats :}

Pourcentages de LS présentant une activité sur la croissance bactérienne.

\begin{tabular}{cc|cc}
\multicolumn{2}{c|}{ Gélose BCP } & \multicolumn{2}{|c}{ Gélose sang } \\
\hline Effet inhibiteur $\left(\mathrm{FI}_{\mathrm{m}}>1\right)$ & $33 \%$ & - Effet inhibiteur $\left(\mathrm{FI}_{\mathrm{m}}>1\right)$ \\
S. aureus & S. aureus & $15 \%$ \\
S. epidermidis & $40 \%$ & S. epidermidis & $10 \%$ \\
E. coli & $\mathbf{1 0}$ & E. coli & $0 \%$ \\
Effet stimulateur $\left(\mathrm{FI}_{\mathrm{m}}<1\right)$ & & - Effet stimulateur $\left(\mathrm{FI}_{\mathrm{m}}<1\right)$ & \\
S. aureus & $0 \%$ & S. aureus & $0 \%$ \\
S. epidermidis. & $10 \%$ & S. epidermidis & $30 \%$ \\
E. coli & $10 \%$ & E. coli & $8 \%$
\end{tabular}


Conclusion :

Nos résultats retrouvent un PAB intermédiaire comparé à ceux de la littérature : globalement, le LS humain a tendance à inhiber les staphylocoques, mais pas $E$. coli. Par contre, nous mettons en évidence qu'il peut avoir un effet stimulateur sur la croissance de $S$. epidermidis et $E$. coli, un même LS pouvant à la fois inhiber la croissance d'une bactérie et stimuler celle d'une autre. Cet effet est majoré sur gélose au sang, contrairement au PAB, ce qui pourrait interférer sur l'interprétation des spermocultures.

1. Rozanski et al.. 1949 ; 2. Rusk et al., 1973 ; 3. Mårdh \& Colleen, 1975.

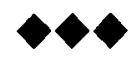

Intérêt des détections immunohistochimiques de la proacrosine et de la protamine 1 dans les prélèvements testiculaires chirurgicaux

\section{MITCHELL ${ }^{1}$, P. MAHE ${ }^{2}$, R.M. SIMINSKI ${ }^{3}$, M.H. GEVAERT ${ }^{3}$, D. ESCALIER ${ }^{4}$, E. HERNANT ${ }^{1}$, F. VIRECOULON ${ }^{1}$, M.C. PEERS ${ }^{1}$, I. KOSCINSKI ${ }^{1}$, J.M. RIGOT ${ }^{2}$, A. DEFOSSEZ ${ }^{1}$. \\ 1: Laboratoire de Spermiologie/Biologie de la Repro- duction, 2: Service d'Andrologie, CHRU de Lille, \\ 3: Laboratoire d'Histologie de la Faculté de Médecine de Lille; ${ }^{4}$ : IFR Biomédicale des Saints Pères, Paris.}

\section{Introduction :}

L'objectif a été d'évaluer l'intérêt des marqueurs des cellules de la lignée germinale en méiose et/ou en post-méiose: la proacrosine et la protamine 1, dans les tissus testiculaires chirurgicaux de patients azoospermes ayant présenté une aplasie ou un arrêt précoce de maturation et pour lesquels l'extraction de spermatozoïdes a été un succès.

\section{Matériel et Méthodes :}

Sur 284 biopsies prélevées entre 1996 et 2001, 33 ont été retenues. 24 étaient histologiquement aplasiques (absence de cellules de la lignée germinale), 9 présentaient un arrêt de maturation précocc (stade spermatogonies ou spermatocytes I). Les tissus testiculaires ont été recoupés en coupes sériées, puis étudiés par la méthode immunohistochimique de marquage de la proacrosine el de la protamine l. Les complexes antigène-anticorps ont été détectés par des immunoglobulines biotinylées, amplifiées par un complcxe streptavidine-biotine-peroxydase révélé par la DAB- $\mathrm{H}_{2} \mathrm{O}_{2}$.

\section{Résultats :}

Les marquages par les anticorps dirigés contre la proacrosine et la protamine 1 ont permis d'identifier des cellules de la lignée germinale dans $45 \%$ (15/33) des biopsies étudiées. Dans $29 \%(7 / 24)$ des tissus aplasiques et $89 \%(8 / 9)$ des tissus avec arrêt précoce de maturation, des spermatides allongées ont été détectées dans un ou plusieurs tubes séminifères.

\section{Conclusion :}

La proacrosine et la protamine 1 sont des marqueurs fiables de la lignée germinale. Ainsi, des spermatides ont pu être mises en évidence dans des tissus histologiquement aplasiques ou en arrêt précoce de maturation. Is améliorent par conséquent de façon non négligeable la sensibilité de la détection histologique des cellules germinales et des spermatides dans les tissus testiculaires des patients azoospermes. Leur utilisation prospective est en cours d'évaluation dans la détection des spermatides dans les tissus testiculaires aplasiques, et dans l'identification des cellules rondes de nature germinale dans les éjaculats azoospermes.

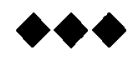

Traitement par une thérapie multidisciplinaire et reconstruction chirurgicale avec l'utilisation d'un expanseur cutané, dans un cas de gangrène de Fournier

\section{G. PASSAVANTI, V. PIZZUTI, F.M. COSTANTINI, M. BRAUZZI* A. TANASI ${ }^{*}$, R. PAOLINI}

Service Urologie-Andrologie ;* Service OTH ; Hôpital

"Misericordia", Grosseto, Italie.

\section{Introduction :}

La Gangrène de Fournier est une cellulite des plans superficiels du périnée qui peut intéresser le scrotum et la paroi abdominale.

\section{Matériel, Méthodes et Résultats}

Un malade diabćtique a été hospitalisé pour l'apparition rapidc d'oedème du périnée et du scrotum; il y avait aussi du tissu nécrosé et hémorragique. La palpation causait un crépitement et de la doulcur. L'état hémodynamique était stable. Après quelques heures nous avons exécuté un large débridement du tissu nécrosé et nous avons réalisé une dérivation urinaire sus-pubienne (Fig. I). Une antibiothéra- 
pie et une oxygénothérapie hyper-bar (OTH) (32 séances OTH à 2.5 ATA-15 m- pour 90 -120min. ; la première phase 2 séances/j. pour 6 jours et ensuite 1 séance/j. pour 20 jours ; durée totale 40 jours) ont été débutées. Après 12 séances thérapeutiques et de pansements locaux, a été réalisé le premier temps opératoirc reconstructif. Il y avait une perte de substance qui intéressait le périnée, le scrotum et la partie proximale de la verge (fig. 2); nous avons placés les deux testicules, dans leur vaginale, dans des logettes sous cutanées inguinales postérieures (fig. 4 et 5) afin d'obtenir assez de tissu tégumentaire pour recouvrir la région scrotale et le périnée ; un lambeau pédiculé de prépuce a été employé pour recouvrir le pénis (fig. 6 et 7 ). Aprés un mois, un expanseur de $6 \times 4 \mathrm{~cm}-25 \mathrm{cc}$ a été placé dessous la peau de la région scrotale pour obtenir assez de tissu et réaliser une loge physiologique (fig. 8 et 9) ; 30 jours après nous y avons placés de nouveau les testicules (fig. 10).

\section{Discussion :}

La gangrène de Fournier est souvent trouvée chez des malades avec une compromission immunitaire (diabète, éthylisme; SIDA) et en général a pour origine un petit traumatisme cutané ou des infections et abcès périurétraux et périanaux.

L'histologie est caractérisée par une cellulite aiguë avec oedème puis une vascularite suivie par une thrombose et enfin une nécrose tissulaire. Les germes identifiés sont aérobies et anaérobies. La mortalité est très élevée, de 20 à $67 \%$. La précocité du traitement améliore la survie et les résultats chirurgicaux. L'OTH améliore le trophisme des tissus peri-lésionnels et protège contre les germes anaérobies et donc doit être associée à la chirurgie et à la thérapie médicamenteuse. La chirurgie se développe sur plusieurs temps: une phase de débridement et de réalisation d'une dérivation sus-pubienne et éventucllement d'une colostomie; puis après détersion, on réalise un temps reconstructif avec des lambeaux cutanés libres ou pédiculés (aussi de prépuce). Si la perte de substance n'est pas excessive, on peut placer les testicules dans une loge sous-cutanée inguinale pour obtenir assez de tissu pour fermer la plaie causée par la maladie. Cette intervention permet une bonne cicatrisation de la peau scroto-périnéale en attendant la reconstruction du scrotum après placement d'un expanseur cutané.

\section{Conclusion :}

Ce cas clinique permet de vérifier qu'un traitement très précoce avec une thérapie multidisciplinaire permet des bons résultats en termes de survie, et que la technique chirurgicale que nous avons employée permet une bonne résolution anatomique de la perte de substance causée par la maladie.
Grossesses évolutives obtenues par ICSI pour deux frères présentant un syndrome des spermatozoïdes décapités

\author{
J. PERRIN,4, G. PORCU2, G. MERCIER3,4, \\ P. BOYER 3 , V. ACHARD 1,4 , J. BANET 4 , \\ M. VASSEROT $4, C$. MELONE $^{3}$, \\ J. SAIAS-MAGNAN1, C. D'ERCOLE5, C. CHAU5, \\ M.R. GUICHAOUA 1,4 . \\ ${ }_{1}^{A}$ P-HM Conception, Laboratoire de Biologie de la \\ Reproduction, 147 bd Baille, 13385 Marseille cedex 05. \\ 2 AP-HM Conception, Centre de Procréation Médicale- \\ ment Assistée, 147 bd Baille, 13385 Marseille cedex 05. \\ ${ }^{3}$ Hôpital Saint Joseph, Laboratoire de Procréation Médi- \\ calement Assistée, 26 bd Louvain, 13285 Marseille cedex \\ 4 Laboratoire d'Histologie et de Biologie de la Reproduc- \\ tion, Faculté de Médecine Timone, 27 bd Jean Moulin, \\ 13385 Marseille cedex 05. \\ 5 Service de Gynécologie Obstétrique, Hôpital Nord, che- \\ min des Bourrelys, 13915 Marseille cedex 20.
}

\section{Introduction :}

Nous rapportons les cas de deux couples dont les hommes sont frères et présentent un syndrome des spermatozoïdes décapités, pour lesquels des grossesses évolutives ont été obtenues après ICSI. Il s'agit à notre connaissance des premiers cas de grossesse évolutive dans le cadre de ce syndrome.

\section{Matériel et Méthodes :}

Les deux frères présentent une oligo-asthénospermie sévère, de nombreux flagelles isolés, une tératospermie totale avec prédominance de têtes isolées et de défaut d'alignement tête-flagelle. L'étude en microscopie électronique a mis en évidence chez les deux paticnts une absence d'alignement tête-flagelle, une tête isoléc ou un flagelle isolé dans $100 \%$ des spermatozoïdes étudiés, ainsi qu'une absence de plaque basale et de fossette d'implantation. L'étude immunocytochimique de la matrice péri-centriolaire par marquage avec anticorps anti-MPM2 est considérée comme normale.

\section{Résultats :}

Chez le premier couple, 4 ovocytes matures ont été injectés au cours de deux cycles d'ICSI ct 4 embryons ont été replacés. Une grossesse évolutive a été obtenue au deuxième cycle, conduisant à la naissance à terme d'une fille de poids normal en bonne santé.

Le second couple a bénćficié de 3 cycles d'ICSI avec injection de 18 ovocytes matures et replacement de 6 embryons. Une grossesse évolutive triple comprenant deux 
jumeaux monozygotes est survenue au troisième replacement embryonnaire. Deux garçons et une fille ont été extraits par césarienne à 30 semaines de grossesse pour hypotrophie fœetale et sont aujourd'hui en bonne santé.

\section{Conclusion :}

Cette étude montre que des grossesses évolutives sont possibles dans le cadre du syndrome des spermatozoïdes décapités. L'origine génétique de ce syndrome semble admise, mais aucun gène n'a encore été identifié. L'hétérogénéité phénotypique observée en microscopie électronique dans les différents cas décrits dans la littérature suggère l'existence d'une hétérogénéité génétique.

Il a été suggéré que ce syndrome résulte d'un défaut de migration caudale des centrioles, d'une absence d'induction de la fossette d'implantation et de la plaque basale permettant l'ancrage du flagelle. Notre étude montre que certaines formes sont compatibles avec la formation normale du sperm aster et un développement embryonnaire correct.

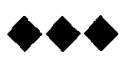

\section{GATA 4 et GATA 6 sont présents dans l'épididy- me murin et modulent in vitro le promoteur de la glutathion peroxydase épididymaire}

\author{
E. PONS, J.R. DREVET \\ Laboratoire Epididyme et Maturation des Gamètes, \\ UMR 6547 CNRS Université Blaise Pascal, 24 av \\ des Landais, 63177 Aubière Cedex, France.
}

\section{Introduction :}

Chez la souris, la glutathion peroxydase 5 est transcrite sclon une localisation stricte dans la tête de l'épididyme du mâle adulte. Nous avions préalablement montré que cette spécificité était sous la dépendance de facteurs de transcription connus, co-localisant avec gpx 5 dans cet organe : le récepteur aux androgènes $(A R)$ et un facteur de la famille Ets, PEA3 (Polyoma Enhancer Activator 3). Ces modulateurs transcriptionnels peuvent rćpondrc d'unc partie au moins de la restriction d'expression de gpx5.

L'analyse consensuelle du promoteur de gpx5 a permis d'identifier de multiples séquences cis de type 5' -WGATAR-3',

cibles potentielles de facteurs de transcription de la famille GATA. Des expériences de retard de migration ont confirmé leur capacité à lier des facteurs nucléaires. Inversement des extraits protéiques nucléaires épididymaires présentent une activité de liaison à l'ADN comparable à celle des facteurs trans de type GATA.

\section{Résultats :}

GATA-1, déjà identifié dans le tractus génital, particulièrement au niveau du testicule, n'a pu être mis en évidence dans l'épididyme murin. Nous avons par contre déterminé que GATA-4 et GATA- 6 étaient exprimés à des niveaux significatifs dans ce tissu notamment, ainsi que dans un choix d'organes sexuels ou somatiques du mâle et de la femelle adulte et au cours du développement. Par co-transfection de ces deux facteurs et d'un rapporteur GFP sous le contrôle de différentes délétions du promoteur de $g p x 5$ en système hétérologue, nous avons montré qu'ils pouvaient en moduler l'activité transcriptionnelle selon un équilibre d'activation/inhibition complexe.

\section{Conclusion :}

Nous proposons ainsi que les facteurs de transcription de la famille GATA, et notamment de la sous-famille 46, puissent être de nouveaux modulateurs de l'activité du promoteur de gpx5 in vivo, et de sa spécificité tissulaire et ontogénétique stricte.

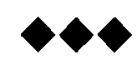

Contrôle spinal de l'érection réflexe par le glutamate : approches morphologique et fonctionnelle chez le rat

O. RAMPIN, J. AIOUN, I. CHAMBILLE, S. GOUGIS, N. JEROME, R. MONNERIE, M.-J. PRUD'HOMME

Laboratoire de Neurobiologie des Fonctions
Végétatives, INRA, F-78352 Jouy-en-Josas, Cedex,

France.
Introduction :

La moelle épinière est le siège des érections réflexes. La stimulation du pénis recrute des fibres sensitives qui gagnent la corne dorsale où elles relaient sur des interneurones. A leur tour, ceux-ci informent les neurones prégan- 
glionnaires autonomes proérectiles de la moelle sacrée par l'intermédiaire de plusieurs autres interneurones. Nous avons cherché à mettre en évidence le rôle du glutamate, puissant neuromédiateur excitateur, dans ce circuit polysynaptique de l'érection réflexe.

\section{Matériel et Méthodes :}

Nous avons testé les effets d'injections intrathécales de NBQX, antagoniste des récepteurs glutamatergiques AMPA, et d'AP5, antagoniste des récepteurs glutamatergiques NMDA, en regard des segments spinaux lombosacrés sur i) l'augmentation de la pression intracaverneuse déclenchée par la stimulation du nerf dorsal du pénis chez le rat anesthésié et ii) le nombre d'érections réflexes déclenchées par la rétraction du fourreau chez le rat éveillé. Nous avons recherché la présence de glutamate, et des sous-types NMDAR1 et GluR1-R4 des récepteurs glutamatergiques à l'aide de techniques d'immunohistochimie sur des coupes de moelle lombosacrée.

\section{Résultats :}

L'injection intrathécale de NBQX comme celle d'AP5 inhibe d'une façon dose-dépendante les augmentations de la pression intracaverneuse déclenchées par la stimulation du nerf dorsal du pénis, ainsi que le nombre des érections réflexes. Nous mettons en évidence le glutamate dans les terminaisons des fibres afférentes primaires entrant dans la corne dorsale. Les sous-types NMDARl et GluR1-GluR3 sont présents dans la corne dorsale, le noyau parasympathique sacré, dans la région autour du canal central, incluant la commissure grise dorsale, et dans la corne ventrale. Les neurones préganlionnaires parasympathiques, marqués par transport axonal rétrograde à partir du nerf pelvien, sont immunoréactifs pour les différents sous-types des récepteurs du glutamate.

\section{Discussion :}

Nous mettons en évidence la participation du glutamate au contrôle spinal de l'érection. Le glutamate agit via des récepteurs AMPA et NMDA. Le glutamate est présent non seulement dans les afférences génitales, mais aussi très certainement dans les interneurones spinaux. Cette hypothèse est suggérée par la présence des récepteurs AMPA et NMDA du glutamate dans les interneurones spinaux contrôlant l'érection. Enfin, si le glutamate est le neuromédiateur principal des afférences sensitives, il doit également participer au contrôle des circuits spinaux par les projections descendantes du tronc cérébral et de l'hypothalamus.

\section{Analyse des mécanismes d'association et de transfert de la glutathion peroxydase GPX5 au spermatozoïde chez le modèle murin}

\author{
H. REJRAJI, P. VERNET, J. DREVET \\ Laboratoire Epididyme et Maturation des Gamètes, \\ UMR 6547 CNRS-Université Blaise Pascal, \\ 24 avenue des Landais, 63177 Aubière, France.
}

\section{Introduction :}

Chez les mammifères, les gamètes issus du testicule ne sont pas fécondants. Leur maturation, (l'aptitude à se mouvoir et à reconnaître l'ovocyte) est réalisée progressivement au cours de leur trajet dans l'épididyme, sous l'influence notamment des sécrétions protéiques épididymaires.

\section{Matériel et Méthodes :}

Nous avons cloné chez la souris, un marqueur androgénodépendant de l'activité sécrétoire de cet organe, la protéine GPX5. Cette protéine produite abondamment et uniquement par les cellules épithéliales de l'épididyme est sécrétée dans le fluide épididymaire et se retrouve associée aux spermatozoïdes tout au long du tractus génital.

\section{Résultats :}

Au cours du transit dans l'épididyme, une diminution de la quantité de GPX5 présente dans le fluide a été observée ainsi qu'une association plus forte de la protéine aux gamètes. Cette association, aussi bien dans la tête que dans la queue épididymaires, confère une protection vis-à-vis des digestions protéolytiques. Cette résistance envers les protéases est retrouvée au niveau de la population de GPX5 présente dans lc fluide épididymaire. D'une façon générale dans les deux segments épididymaires, ces protections sont abolies lors de traitements physico-chimiques stringents tels que les ultrasons ou un $\mathrm{pH}$ acide. L'utilisation d'enzymes lipolytiques permet également la libération de GPX5. Ainsi, dans la lumière de l'épididyme, GPX5 est retrouvée dans trois localisations différentes. GPX5 existe sous une forme soluble dans le fluide, liées aux gamètes et associées à des structures riches en lipides. Ces dernières structures ont été isolées et caractérisées notamment en microscopie électronique et par anisotropie de fluorescence. Elles sont le résultat de mécanismes de sécrétion apocrine de l'épithélium épididymaire, se présentant sous la forme de vésicules hétérogènes en taille et en structure. Ces "épididymosomes" possèdent une composition et une dynamique membranaire relativement stable quelque soit le stade de maturation. 
Conclusion :

Ces vésicules pourraient constituer des intermédiaires importants dans le transfert de molécules aux spermatozoïdes (phospholipides, cholestérol, protéines particulières telles que (GPX5), contrôlant ainsi l'acquisition du pouvoir fécondant des gamètes mâles, et pourraient être exploitées à des fins thérapeutiques ou contraceptives.

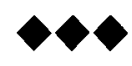

\section{Altération de la spermatogénèse chez les hom- mes présentant une agénésie bilatérale des canaux déférents}

\author{
P. MAHE ${ }^{1}$, F. MARCELLI ${ }^{1}$, B. SOULEZ ${ }^{2}$, \\ J-C. HERBAUT ${ }^{3}$, E. HERMAND ${ }^{4}$, J-M. RIGOT ${ }^{1}$ \\ ${ }^{1}$ Service d'Andrologie, 2 Service de Biologie de la \\ Reproduction, 4 Laboratoire d'Histologie \\ Fonctionnelle, C.H.R.U., ${ }^{3}$ EPARP, Lille.
}

\section{Introduction :}

L'azoospermie en cas d'agénésie bilatérale des canaux déférents $(A B C D)$ est supposée être d'origine excrétoire. Nous avons évalué la spermatogenèse par histologie testiculaire chez des hommes azoospermiques en rapport avec une $\mathrm{ABCD}$.

\section{Matériel et Méthodes :}

55 hommes présentant une $\mathrm{ABCD}$ avec désir de grossesse ont été pris en charge par prélèvement chirurgical de spermatozoïdes après évaluation pneumologique et recherche de mutations du gène CFTR.

\section{Résultats :}

Des spermatozoïdes ont été retrouvé dans tous les cas. 17 fois par prélèvement épididymaire seul ; dans 38 cas une biopsie testiculaire a été réalisée dont l'examen histologique montrait :

$\begin{array}{lr}\text { Spermatogenèse normale }: & 20 \text { cas } \\ \text { Blocage de maturation }: \ldots . & 4 \text { cas } \\ \text { Aplasie germinale }: \ldots . . . . & 1 \text { cas } \\ \text { Hypospermatogenèse }: \ldots . . & 13 \text { cas } \\ \text { Sclérose tubulaire : ......... } & 0 \text { cas }\end{array}$

Conclusion :

Cette étude montre que les hommes présentant une ABCD n'ont pas tous une spermatogenèse normale : 18 cas de troubles de la spermatogenèse retrouvé soit $47 \%$ des biopsies réalisées et $32,7 \%$ des $\mathrm{ABCD}$ de notre série. Le facteur obstructif ne doit donc pas faire négliger la recherche associée d'une cause génétique ou testiculaire d'altération de la spermatogenèse.

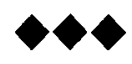

\section{Prélèvements chirurgicaux de spermatozoïdes en cas d'azoospermie : Résultats de six années d'activité}

\author{
P. MAHE ${ }^{1}$, F. MARCELLI ${ }^{1}$, B. SOULEZ 2 , \\ J-C. HERBAUT ${ }^{3}$, J-M. RIGOT ${ }^{1}$ \\ ${ }^{1}$ Service d'Andrologie, 2 Service de Biologie de la \\ Reproduction, ${ }^{3}$ EPARP, Lille.
}

\section{Introduction :}

L'injection intra cytoplasmique de spermatozoïdes (ICSI) avec sperme chirurgical en cas d'azoospermie est de pratique courante dans les centres de procréation médicalement assistée (PMA). Nous avons voulu faire le bilan de notre activité depuis 1996.

\section{Matériel et Méthodes :}

Les résultats des prélèvements de tous les patients azoospermiques pris en charge de janv. 1996 à déc. 2001 ont été rapportés et classés selon l'origine de l'azoospermie (taux de FSH).

\section{Résultats :}

\begin{tabular}{|c|c|c|c|c|c|c|c|c|}
\hline & \multicolumn{2}{|c|}{$1996-1997$} & \multicolumn{2}{|c|}{ 1998-1999 } & \multicolumn{2}{|c|}{$2000-2001$} & \multicolumn{2}{|c|}{ Total } \\
\hline & $\begin{array}{l}\text { Plvt. } \\
+\end{array}$ & Plvt & $\begin{array}{l}\text { Plvt. } \\
+\end{array}$ & Plvt & $\begin{array}{l}\text { Plvt. } \\
+\end{array}$ & $\begin{array}{l}\text { Plvt } \\
-\end{array}$ & $\begin{array}{l}\text { Plvt. } \\
+\end{array}$ & $\begin{array}{l}\text { Plvt. } \\
- \\
\end{array}$ \\
\hline $\begin{array}{l}\text { Az. } \\
\text { Sécrétoires }\end{array}$ & $\begin{array}{l}19 / 46 \\
41 \%\end{array}$ & $27 / 46$ & $\begin{array}{l}26 / 58 \\
45 \%\end{array}$ & $32 / 58$ & $\begin{array}{l}46 / 79 \\
58 \%\end{array}$ & $33 / 79$ & $\begin{array}{l}91 / 183 \\
50 \% \\
\end{array}$ & \\
\hline $\begin{array}{l}\text { Az. } \\
\text { Excrétoires }\end{array}$ & $\begin{array}{l}50 / 57 \\
88 \%\end{array}$ & $7 / 55$ & $\begin{array}{l}36 / 38 \\
95 \%\end{array}$ & $2 / 38$ & $\begin{array}{l}45 / 54 \\
83 \%\end{array}$ & $9 / 54$ & $\begin{array}{l}131 / 149 \\
88 \% \\
\end{array}$ & \\
\hline A.B.C.D. & $\begin{array}{l}19 / 19 \\
100 \%\end{array}$ & & $\begin{array}{l}20 / 20 \\
100 \%\end{array}$ & & $\begin{array}{l}16 / 16 \\
100 \%\end{array}$ & & $\begin{array}{l}55 / 55 \\
100 \%\end{array}$ & \\
\hline $\begin{array}{l}\text { An- } \\
\text { éiaculations }\end{array}$ & $\begin{array}{l}8 / 9 \\
89 \%\end{array}$ & & $\begin{array}{l}3 / 3 \\
100 \% \\
\end{array}$ & & $\begin{array}{l}3 / 3 \\
100 \% \\
\end{array}$ & & $\begin{array}{l}14 / 15 \\
93 \%\end{array}$ & \\
\hline Total & $\begin{array}{l}96 / 131 \\
73 \%\end{array}$ & & $\begin{array}{l}85 / 119 \\
71 \%\end{array}$ & & $\begin{array}{l}110 / 152 \\
72 \% \\
\end{array}$ & & $\begin{array}{l}291 / 402 \\
72 \% \\
\end{array}$ & \\
\hline
\end{tabular}




\section{Conclusion :}

Dans notre expérience il est possible de retrouver des spermatozoïdes dans $72 \%$ des cas d'azoospermie. Le taux de recueil est corrélé au taux de FSH. On note une stabilité dans le temps de l'extraction dans les Azoospermies excrétoires et une augmentation dans les Azoospermies sécrétoires.

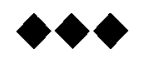

\section{Apport de l'histologie testiculaire dans la prise en charge de l'azoospermie par prélèvement chirurgical de spermatozoïdes}

\author{
P. MAHE ${ }^{1}$, F. MARCELLI ${ }^{1}$, B. SOULEZ 2 , \\ J-C. HERBAUT ${ }^{3}$, E. HERMAND ${ }^{4}$, J-M. RIGOT ${ }^{1}$ \\ ${ }^{1}$ Service d'Andrologie, ${ }^{2}$ Service de Biologie de la \\ Reproduction, ${ }^{4}$ Laboratoire d'Histologie \\ Fonctionnelle, C.H.R.U., ${ }^{3}$ EPARP, Lille.
}

\section{Introduction :}

Le taux de recueil de spermatozoïdes sur biopsie testiculaire est supérieur si le volume biopsié est plus élevé. Or, la valeur prédictive de l'histologie de la présence de spermatozoïdes n'est pas absolue et la découverte fortuite de pathologies malignes est rare. Doit-on donc continuer l'analyse histologique des biopsies testiculaires dans cette indication?

\section{Matériel et Méthodes :}

Etude des résultats des prélèvements chirurgicaux de spermatozoïdes et histologiques de 402 patients azoospermiques.

\section{Résultats :}

23\% de spermatozoïdes retrouvés en cas d'aplasie germinale, 1 cancer détecté (Tumeur de Leydig) sur 402 hommes opérés, alors que l'échographie testiculaire préopératoire était normale.

\begin{tabular}{lccc}
\hline & $\begin{array}{c}\text { Prélèvement } \\
(\mathrm{n}=291)\end{array}$ & $\begin{array}{c}\text { Prélèvement - } \\
(\mathrm{n}=111)\end{array}$ & $\begin{array}{c}\text { Total } \\
(\mathrm{n}=402)\end{array}$ \\
\hline Normal & $96 \%(46)$ & $4 \%(2)$ & 48 \\
Hypoplasie modérée & $100 \%(80)$ & 0 & 80 \\
Hypoplasie sévère & $92 \%(68)$ & $\mathbf{8} \%(6)$ & 74 \\
Blocage de maturation & $75 \%(21)$ & $25 \%(7)$ & 28 \\
Aplasie & $23 \%(28)$ & $77 \%(96)$ & 124 \\
Non fait & $100 \%(48)$ & 0 & 48 \\
\hline
\end{tabular}

Conclusion :

L'histologie testiculaire dans cette indication paraît peu intéressante par sa faible valeur prédictive de la présence de spermatozoïdes et le faible taux de découverte fortuite de néoplasies testiculaires. Néanmoins, la fécondance, moins bonne dans notre expérience, des spermatozoïdes d'origine sécrétoire et les conséquences médico-légales de l'absence de documents histologiques, ne permettent pas de se passer de cette examen actuellement.

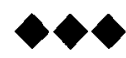

\section{Du spermatocyte au spermatozoïde : anomalies chromosomiques et hypospermatogenèse}

\author{
N. RIVES ${ }^{1}$, A. BORDES ${ }^{1}$, J-P. MILAZZO' 1 , M-O. \\ NORTH $^{1}$, L. SIBERT ${ }^{2}$, N. MOUSSET-SIMEON ${ }^{1}$, \\ B. MACE 1 \\ ${ }^{1}$ Laboratoire de Biologie de la Reproduction, \\ ${ }^{2}$ Service d'Urologie, CHU Charles Nicolle \\ 76031 Rouen
}

\section{Introduction :}

Chez l'homme, dans le contexte d'une spermatogenèse déficiente, on peut envisager l'existence d'une relation entre anomalies chromosomiques spermatiques et déplétion en gamètes matures haploïdes. Ces anomalies chromosomiques spermatiques peuvent être générées au cours des divisions des spermatogonies et/ou au cours de la méiose. Les spermatogonies ou spermatocytes porteurs de ces anomalies peuvent soit s'arrêter au cours du cycle cellulaire, soit poursuivre la spermatogenèse en produisant des spermatozoïdes porteurs d'anomalies chromosomiques. Notre étude se proposait d'établir une relation entre les anomalies chromosomiques observées dans les cellules germinales haploïdes, les altérations de la méiose et l'augmentation de l'indice apoptotique des cellules germinales.

\section{Matériel et Méthodes :}

Cette étude porte sur un échantillon de 17 patients, âgés de 24 à 52 ans, consultant dans le Laboratoire de Biologie de la Reproduction pour exploration d'une infcrtilité primaire ou secondaire : 12 patients présentent un défaut partiel (oligoasthénotératozoospermie) ou complet (azoospermie 
sécrétoire) de la production des spermatozoïdes (HYP), 5 patients présentent une azoospermie excrétoire par obstruction congénitale (Absence Bilatérale Congénitale des Canaux Délérents ou A.B.C.D) ou acquise (vasectomie) des voies excrétrices spermatiques (AE). Chaque patient a bénéficié d'une exploration chirurgicale testiculaire bilatérale au cours de laquelle une biopsie a été réalisée. Une analyse histologique et une évaluation des anomalies méiotiques au stade pachytène par coloration au Giemsa et par hybridation in situ en fluorescence (chromosomes X, Y, 13 et 21) ont été effectuées sur chaque prélèvement. Cette première approche a été complétée par une évaluation de la fréquence des anomalies chromosomiques observées dans les cellules germinales haploïdes en suspension par couplage de l'immunocytochimie à l'aide de l'anticorps Mab 4D4 reconnaissant la proacrosine et l'hybridation in situ en fluorescence (chromosomes $X, Y$ et 18). Enfin, la fragmentation de l'ADN des cellules germinales a été détectée par la méthode TUNEL sur des coupes testiculaires.

\section{Résultats :}

L'étude des altérations de la méiose par les techniques de cytogénétique standard a révélé que 6 patients parmi les 12 du groupe HYP présentaient des anomalies du stade pachytène (4 présentaient des asynapsis interstitiels, 2 des anomalies de la vésicule sexuelle). L'hybridation in situ en fluorescence sur préparations chromosomiques de noyaux au stade pachytène a démontré une augmentation non significative de l'association des chromosomes 13 et 21 avec la vésicule sexuelle chez les patients du groupe HYP par rapport au groupe AE (HYP : $6,5 \%$ et $5,7 \%$ vs AE: $4,7 \%$ et $5,1 \%$ respectivement). L'analyse des cellules germinales par hybridation in situ en fluorescence et immunocytochimie à l'aide de l'anticorps Mab 4D4 est en faveur d'une augmentation significative du taux d'aneuploïdie des chromosomes sexuels dans les cellules haploïdes chez les patients du groupe HYP par rapport aux patients du groupe $\mathrm{AE}(0,01 \leq \mathrm{p} \leq 0,02)$. Enfin, la mesure de la fragmentation de l'ADN met en évidence une augmentation significative de l'indice apoptotique des spermatogonies et des spermatocytes des patients du groupe HYP ( $p=0,035$ et $p=0,035$ respectivement).

\section{Conclusion :}

Ces résultats confirment que les cellules germinales altérées lors de la méiose sont soit à l'origine de gamètes aneuplö̈des soit éliminées par un processus apoptotique sous la dépendance probable de mécanismes mettant en jeu les points de contrôle intra-méiotiques du cycle cellulaire.

\section{Investigation de la spermatogenèse par la cytométrie en flux séminale chez des hommes inféconds}

\author{
Y. SOFFER 1,2, M. WALD 1 , R. GOLAN ${ }^{1}$, \\ L. SHOCHAT ${ }^{1}$, A. RAZIEL, R. RON-EL et \\ L.M. LEWIN ${ }^{1}$
}

\author{
${ }^{1}$ Département de Biochimie Clinique, Faculté de \\ Médecine Sackler, Tel-Aviv \\ 1 Institut de fertilité masculine et Unité de FIV, CHU \\ Assaf Harofe, Zerifin. \\ 1,2 Université de Tel-Aviv, Tel-Aviv, Israël.
}

\section{Introduction :}

Dans les tubules séminifères, les spermatogonies diploïdes donnent, par une série de métamorphoses, des spermatocytes primaires tétraploïdes, des spermatocytes secondaires diploïdes, des spermatides haploïdes et finalement des spermatozoïdes matures condensés. Toutes ces cellules finissent par se retrouver, en proportions variées, dans l'éjaculât où elles ont pu être identifiées (Hacker-Klom et col, Human Reproduction, 14:2506-12). La cytométrie en flux du sperme permettrait donc chez des hommes inféconds d'obtenir un aperçu de leur spermatogenèse bien avant une intervention testiculaire (TESE) pour micro injection (ICSI). Le but de ce travail a été d'obtenir des profils cytométriques d'éjaculâts et d'évaluer leur intérêt clinique dans le bilan des infécondités et surtout des azoospermies non obstructives (NOA) avant TESE et ICSI.

\section{Matériel et Méthodes :}

La cytométrie en flux du sperme avec marquage de l'ADN à l'iodure de propidium a été faite avec un "FacSort" chez des sujets oligo-térato-asthénozoospermiques (OTA) ou azoospermiques (NOA) ainsi que chez des donneurs normaux pour contrôle. En plus de l'analyse de routine du sperme, une recherche minutieuse de spermatozoïdes après centrifugation (ESP) a été pratiquée si nécessaire. L'ICSI a été fait selon les cas avec ou sans TESE. Les cellules séminales ont été stockées après centrifugation à $-80^{\circ} \mathrm{C}$ en milieu TNE jusqu'à l'cxamen. Le triage cytométrique a été analysé sur graphique bi-dimensionnel avec le logiciel WinMDI v2.8 (http://facs.script.educ/). La ploïdie de la chromatine et son degré de condensation ont permis d'identifier et de quantifier les différents types de cellules trouvées dans l'éjaculât. Ce profil cytométrique a été comparé aux variables du sperme, à l'ESP et à l'histologie testiculaire (TESE). 


\section{Résultats :}

Divers types de cellules ont été relevés en proportions variables : 1-Diploides $(D)$ incluant des spermatogonies, des cellules de Sertoli ou du tractus génital et des leucocytes ; 2-Tétraploïdes ( $T$ ) représentant des spermatocytes en méiose ; 3-Haploïdes non-condensées (Hnc), des spermatides allongées ; 4- Haploïdes condensées (Hc), des spermatozoïdes matures. Dans 6 éjaculâts normaux, presque toutes les cellules triées $(98 \%)$ ont été relevées dans la région $H c$. Chez 84 sujets inféconds, le pourcentage de cellules $H c$ exprimé par la médiane (et les quartiles $75 \%$ $25 \%)$ était nettement inférieur : $85 \%(100-60)$ avec des pourcentages accrus de cellules $H n c$ 9\% (18.5-2.5), D $2.5 \%(8-1)$ et $T 0.2 \%(2-0)$ en corrélation avec les variables du sperme. Chez 38 azoospermiques, une coordination excellente a été trouvée entre la découverte de spermatozoïdes à l'ESP et le profil cytométrique. Le pic $H c$ était absent ou très bas dans les cas négatifs à l'ESP. Une coordination semblable a été trouvée avec l'histologie testiculaire dans 14 cas.

\section{Conclusion :}

La cytométrie en flux de l'éjaculât avec marquage de la chromatine permet, avant la biopsie testiculaire, une bonne évaluation des troubles de la spermatogénèse. Dans les azoospermies non obstructives graves, négatives à l'ESP, elle peut aider le clinicien dans le choix du geste thérapeutique adéquat.

\section{$\bullet \bullet$}

Hormone anti-müllérienne séminale : un possible marqueur de la spermatogenèse dans les azoospermies non-obstructives

\author{
E. THIBAULT, S. MIRALLIE, M. JEAN, \\ O. BOUCHOT, P. BARRIERE
}

Hôpital Mère et Enfant, 7 quai Moncousu 44093 Nantes Cedex 01 France

\section{Introduction :}

Il reste très difficile de fixer lc pronostic d'unc biopsic testiculaire pour des patients présentant une azoospermie nonobstructive. Suivie d'une ICSI, elle représente la seule thé- rapeutique homologue envisageable dans ce cadre. Les marqueurs cliniques (volume testiculaire) et sériques actuels (FSH, Inhibine B) ne semblent pas discriminants (Tournaye 1997, Kim 1997, Seo 2001). L'Hormone AntiMüllérienne est spécifiquement produite par les cellules de Sertoli et sa présence dans le liquide séminal semblerait être corrélée à une spermatogenèse persistante (Fusijawa, Hum. Reprod 2002). Cette propriété pourrait être appliquée à l'évaluation de la probabilité de retrouver des spermatozoïdes dans les biopsies testiculaires de patients azoospermes non-obstructifs, ceci pour fixer au mieux les indications de la biopsie (Fénichel, Hum. Reprod. 1999).

\section{Matériel et Méthodes :}

Nous avons quantifié l'AMH séminale de 14 donneurs fertiles (Groupe I), 24 azoospermes non-obstructifs ayant bénéficié d'une biopsie testiculaire (Groupe IIa : biopsie positive/ Groupe IIb : biopsie négative) et 6 azoospermes obstructifs (Groupe III). Le dosage fait appel à une méthode de type ELISA (Beckman Coulter, Marseille, France).

\section{Résultats :}

Les résultats globaux sont présentés tableau 1. Le tableau 2 montre la distribution de l'AMH séminale en fonction des groupes. Les valeurs obtenues dans le groupe II ont été comparées au résultat de la biopsie testiculaire : sur 15 patients présentant une valeur d'AMH supérieure au seuil de sensibilité $(0.7 \mathrm{pmol} / \mathrm{L}), 10$ ont eu une biopsie positive (Groupe IIb) alors que sur les 9 patients dont l'AMH est indétectable, 7 ont eu une biopsie négative (Group IIb). Dans le groupe III, tous les patients présentent une AMH indétectable, confirmant la probable origine testiculaire de l'hormone.

Tableau 1 : AMH séminale : résultats globaux (moyennes \pm écart-types).

$$
\text { Groupe I Groupes IIa + IIb Groupe III }
$$

$\begin{array}{llll}\text { n } & 14 & 24 & 6\end{array}$

AMH (pmol/L) $122.2 \pm 154.5 \quad 5.2 \pm 16.1 \quad 0$
Range
$(0-578)$
$(0-79.5)$

\section{Conclusion :}

Nous avons mesuré la FSH sérique pour chacun des patients azoospermes non-obstructifs du groupe II. La moyenne obtenue pour le groupe IIa (biopsies positives) est de $15.6 \pm 10.9 \mathrm{UI} / \mathrm{L}$ c'est à dire supérieure à celle obtenue pour le groupe IIb (biopsies négatives) soit 13.5 \pm 8.0 UI/L. Dans cette étude nous retrouvons $42 \%$ de biopsies positives quand la FSH est supérieure à 12UL/L. Nous confirmons l'absence de corrélation entre FSH et AMH $(r=-0.154)$.

La valeur prédictive positive de l'AMH séminale per- 
mettant d'évaluer les chances de retrouver des spermatozoïdes lors d'une biopsie testiculaire est de $66.7 \%$ et la valeur prédictive négative de $77.8 \%$. La sensibilité et la spécificité de la technique utilisée sont respectivement de $83.3 \%$ et $58.3 \%$.

L'AMH, en association avec d'autres paramètres tels que le volume testiculaire, la FSH, l'Inhibine B et les d'affiner la valeur pronostique du bilan d'infertilité dans les azoospermies non-obstructives afin d'éviter une biopsie inutile ou de poser l'indication d'une seconde biopsie.

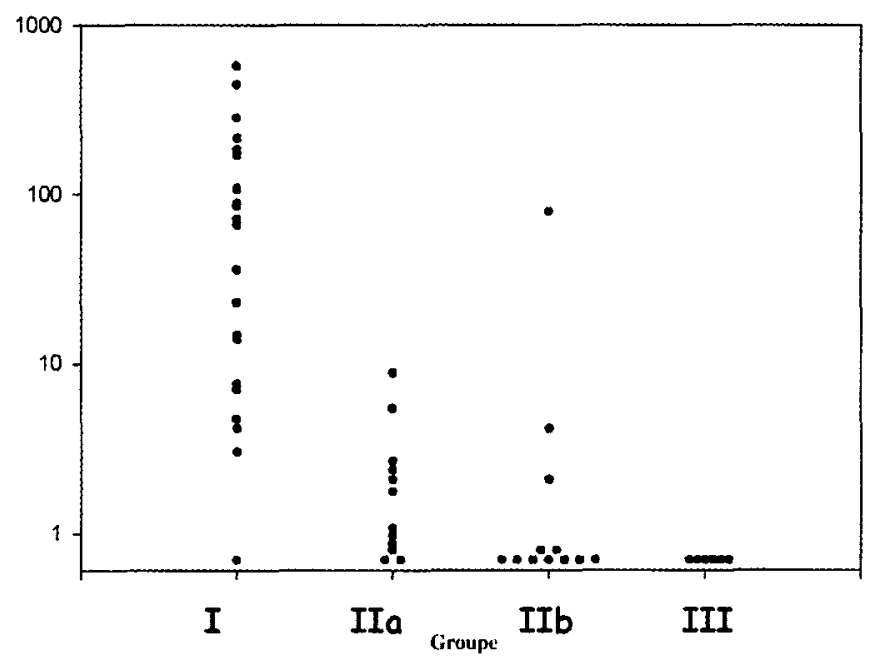

Tableau 2 : distribution des valeurs d'AMH séminale en fonction des groupes. microdélétions du chromosome $Y$ pourrait permettre

young men. Patients with testicular cancer have an excellent survival rate, and fertility is one of the main concerns of survivors. We investigated fertility status after testis cancer treatment by conducting a large study in patients diagnosed with testicular cancer.

Materials and Methods :

We enrolled 506 consecutive patients with testicular cancer (1979 to 1999) at health facilities of the French Midi-Pyrenees region. Testis tumours were classified according to the Royal Marsden Hospital Classification. We assessed fertility status by means of a mailed standardized questionnaire on reproductive events occurring before and after cancer treatment (participation rate of $95 \%$ ).

Results :

Before testis cancer, 242/446 (54.3\%) patients had tried to get their partners pregnant, and $218(90.1 \%)$ had succeeded. After testis cancer treatment, $170 / 413(41.2 \%)$ men tried to get their partner pregnant, and $105 / 170(61.8 \%)$ succeeded.

By comparing testis cancer treatments we observed that radiotherapy, a combination of chemotherapy + radiotherapy in cases of relapse or advanced histological stage, and surgery resection of residual retroperitoneal tumour mass had much more deleterious effects on fecundability than did chemotherapy treatment alone. Cumulative conception rates (log rank test) for patients treated by radiotherapy were significantly lower than those for patients treated by chemotherapy.

\section{Conclusion :}

The major finding of our study was the low fertility rate observed in couples in which the male partner was treated for testis cancer, and his may be largely attributed to treatment-induced damage to the gonads, especially following radiotherapy.

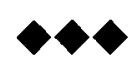

E. HUYGHE, T. MATSUDA, M. DAUDIN, J-M. BACHAUD , C. CHEVREAU, P. PLANTE, R. MIEUSSET, L. BUJAN, P. THONNEAU

Human Fertility Research Group, Hôpital La Grave, Toulouse, France [THONNEAU.P@CHU-TOULOUSE.FR]

\section{Introduction :}

Testicular cancer is the most common malignancy in

\section{Increasing incidence of testicular cancer world- wide : a review}

\author{
E. HUYGHE, T. MATSUDA, P.F. THONNEAU
}

Human Fertility Research Group, Urology and Andrology Unit, La Grave Hospital, Toulouse, France.thonneau.p@chu-toulouse.fr 


\section{Introduction :}

Testicular cancer (TC) is the most common malignancy in men aged 20 to 34 years. Numcrous publications have reported an increase in the incidence of testis cancer over the past forty years, with substantial differences between countries.

\section{Materials and Methods :}

To evaluate worldwide variations in testicular cancer incidence and compare trends in different regions in the world, we reviewed 441 papers yielded by a MEDLINE search using the keywords "testis / testicular", "cancer / tumor" and "incidence", and published between 1980 and 2002. From these, we selected only articles devoted to testis cancer incidence and among them only the most recent papers from each country or region. Nevertheless, articles using the same data base but giving new and additional information (for example differences between ethnic groups or controversial explanations for trends) were also retained. Finally, we selected 30 articles and analyzed their methodological approach and main results.

\section{Results :}

World-wide, we observed a clear trend towards an increase in TC incidence in the past 30 years in a large majority of industrialized countries, in North America, Europe and also Oceania. Nevertheless, surprising differences in incidence rates were seen between neighbouring countries (Finland 2.5/100,000 versus Denmark 9.2/100,000), and also between regions of the same country (from 2.8 to $7.9 / 100,000$, according to various regional French registers). In addition, substantial differences in TC incidence and trends have been observed between ethnic groups. The increase in TC incidence is significantly associated with a birth cohort effect in the USA and also in European countries. So far, cxcept for cryptorchidism, no evident TC risk factor has been clearly demonstrated although the "environmental hypothesis with a key role of endocrine disruptors" has been put forward by several authors.

\section{Conclusion :}

Such a recent increase in TC rate in most industrialized countries should lead urologists and andrologists to give more attention to testicular cancer symptoms in adolescents and young adults. In a public health perspective, further research using cases collected through national and regional population-based registers and case-control studies must be strongly encouraged if we wish to be able to assess future trends in TC incidence rates and also to identify risk factors.
Localisation des protéines phosphorylées sur les résidus tyrosine dans le spermatozoïde humain au cours de la capacitation et après attachement à la zone pellucide

G. LEPPENS-LUISIER ${ }^{1}$, D. SAKKAS ${ }^{2}$, H. LUCAS ${ }^{1}$, C. DE VANTERY ${ }^{1}$, D. CHARDONNENS ${ }^{1}$, A. CAMPANA ${ }^{1}$, D.R. FRANKEN ${ }^{3}$, F. URNER $^{1}$

${ }^{1}$ Clinique de Stérilité et d'Endocrinologie Gynécologique, Département de Gynécologie et d'Obstétrique, Hôpitaux Universitaires de Genève, 1211 Genève 14, Suisse, ${ }^{2}$ Department of Obstetrics and Gynecology, Yale University School of Medicine, New Haven, CT 06520, USA, and ${ }^{3}$ Department of Obstetrics and Gynecology, Reproductive Biology Unit, University of Stellenbosch and Tygerberg Hospital, Tygerberg, South Africa

\section{Introduction :}

La capacitation du spermatozoïde représente une étape nécessaire à la fécondation. Un des éléments clés de la capacitation est la phosphorylation des résidus tyrosines de certaines protéines présentes dans le spermatozoïde. L'objectif de cette étude était de localiser les protéines phosphorylées dans les différents compartiments du spermatozoïde pendant la capacitation et après attachement à la zone pellucide. La phosphorylation de ces protéines a également été mise en relation avec le pouvoir fécondant des spermatozoïde, déterminé lors d'un traitement par Fécondation In Vitro (FIV).

\section{Matériel et Méthodes :}

Quarante et un couples candidats à la FIV ont été inclus dans cette ćtude. Pour chaque couple, le sperme utilisé pour la FIV a également été utilisé pour la localisation des phosphotyrosines. Des expériences d'attachement de spermatozoïdes à la zone pellucide humaine ont été réalisées avec des échantillons fournis par quatre donneurs différents. Les zones pellucides provenaient d'ovocytes humains en prophase I conservés dans une solution hypersaline. Les résidus tyrosines phosphorylés (phosphotyrosines) ont été mis en évidence par immunofluorescence indirecte.

\section{Résultats :}

Les phosphotyrosines ont été détectées surtout dans la pièce principale, alors que relativement peu de spermatozoïdes présentaient un marquage au niveau du segment équatorial, de l'acrosome entier et du cou. Le pourcentage 
de spermatozoïdes avec une pièce principale positive augmentait significativement avec la capacitation, mais quand il restait inférieur à $7 \%$ le taux de fécondation était diminué. Après attachement à la zone pellucide, la proportion de spermatozoïdes présentant des phosphotyrosines simultanément dans la pièce principale ct dans le cou était significativement plus élevée.

\section{Conclusion :}

L'augmentation des phosphotyrosines au niveau de la pièce principale est probablement liée à l'hyperactivation de la mobilité tandis que la signification de la phosphorylation de protéines sur des résidus tyrosines dans le cou reste à définir.

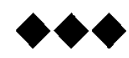

\section{Les cellules testiculaires d'hommes infertiles expriment HLA-G; cette expression corrèle avec le score histologique et le taux d'inhibine $B$}

\author{
B. ZORN 1 , A. IHAN 2 , A. KOPITAR ${ }^{2}$, J. ?INKOVEC 1 \\ ${ }^{1}$ Centre d'andrologie, Clinique gynécologique \\ universitaire, Ljubljana, Slovénie \\ 2 Institut de microbiologie et d'immunologie, Faculté \\ de médecine, Ljubljana, Slovénie
}

\section{Introduction :}

Classiquement, il est admis que dans le testicule les réponses antigéniques contre les spermatozoïdes en relation avec l'immunité cellulaire sont bloquées : en effet, les spermatozoïdes manqueraient d'antigènes du système d'histocompatbilité (HLA), antigènes nécessaires aux réponses des lymphocytes CD4 et CD8. Pour certains auteurs, cependant, les spermatozoïdes ne seraient pas dépouvus de tels antigènes. Le but de cette étude a été d'analyser en cytométrie de flux le tissu testiculaire d'hommes infertiles, auparavant mis en contact avec des anticorps anti HLA-G.

\section{Matériel et Méthodes :}

Seize hommes (âge moyen : $33 \pm 5$ ans) azoospermiques ont bénéficié d'une biopsie testiculaire dans le cadre du programme d'ICSI. L'analyse histologique consistait en hypoplasie germinale $(n=11)$, arrêt de la maturation $(n=3)$, et syndrome de Sertoli-cell only $(n=3)$. Le matériel biopsique a été rincé avec le milieu de culture RPMI et mécaniquement dispersé à l'aide de Medimachine (Becton Dickinson, Mountain View, CA). Les anticorps monoclonaux de souris anti HLA-G MEM-G1 ont été obtenus gracieusement auprès de Philippe Le Bouteiller (INSERM U395, France). L'analyse en cytométrie de flux a été réalisé en utilisant le FACSort (Becton Dickinson, Mountain View, CA) qui distribuait les cellules en fonction de l'intensité de leur fluorescence. L'expression de l'HLA-G a été corrélée au volume testiculaire, au taux plasmatique d'inhibine B et au score histologique.

\section{Résultats :}

L'expression d'HLA-G corrélait négativement avec le volume testiculaire et le taux d'inhibine $B$. Après détermination de l'expression de HLA-G par les cellules testiculaires, nous avons réparti les patients en trois groupes en fonction du score histologique de Johnsen (J): ceux avec hypoplasie germinale $(\mathrm{J}=8,9)$, avec arrêt de la maturation $(\mathrm{J}=5,6)$, et ceux avec syndrome de Sertoli cell-only $(\mathrm{J}=1$, 2).
Score de Johnsen (n)

$\mathrm{J}=1,2(\mathrm{n}=3)$

$J=5,6(n=3)$

$\mathrm{J}=8,9(\mathrm{n}=10)$
Expression de HLA-G

431,3

228,7

87,0
Dans les trois groupes histologiques, nous avons démontré des différences significatives dans l'expression de HLA-G par les suspensions de cellules testiculaires analysées en cytométrie de flux. L'intensite la plus forte a été observée dans les specimen issus de syndrome de Sertoli cell-only tandis que la basse était exprimée par les cellules de tubules à spermatogénèse complète.

\section{Conclusion :}

Dans cette étude, l'expression de HLA-G par les cellules testiculaires semble être un important paramètre, associé aux caratéristiques cliniques, histologiques et hormonales des hommes infertiles.

L'implication primaire ou secondaire du système HLAG dans les cellules testiculaires sera discutée en fonction de la pathologie testiculaire suspectée. 


\section{Hypogonadismes hypogonadotrophiques : démarche thérapeutique nouvelle à l'orée de l'ICSI}

B. ZORN1, M. PFEIFER2, H. MEDEN-VRTOVEC1. 1 Centre d'andrologie, Clinique gynécologique universitaire, Ljubljana, Slovénie.

2Service d'endocrinologie, Centre clinique universitaire, Ljubljana, Slovénie.

Introduction :

Les hypogonadismes hypogonadotrophiques $(\mathrm{HH})$, de causes variées, sont une cause curable d'infertilité. Le traitement classique à base de gonadotrophines et d'accouplement est cependant long, d'au moins 18 mois et aléatoire. Nous avons eu l'idée d'y associer l'ICSI.

Méthodes et résultats :

Nous présentons 3 cas d'HH qui après traitement hormonal ont bénéficié de l'ICSI (Tableau 1).
Discussion / Conclusion :

Classiquement, le traitement des hypogonadismes hypogonadotrophiques en vue de grossesse comporte l'administration d'hCG pendant 3 à 6 mois puis l'association hCG-HMG. Dans 65\% des cas, à 18 mois de traitement le volume testiculaire double et le spermogramme comporte $1,5 \times 10^{6}$ spermatozoïdes $/ \mathrm{ml}$. Un bon volume testiculaire, la survenue de l'HH après la puberté chez un homme plus âgé, sans autre facteur de stérilité (en particulier chez la partenaire) et l'absence de panhypopituitarisme sont les facteurs de bonne réponse au traitement hormonal (Liu et al., Hum. Reprod. 2002). Nous y ajouterons un taux plasmatique de base de l'inhibine $B$ élevé.

En ICSI, l'utilisation de spermatozoïdes éjaculés frais ou congelés-décongelés dès le 12ème mois de traitement hormonal permet l'obtention de grossesses. Les spermatozoïdes en nombre et de mobilité limités ont cependant un haut pouvoir fécondant. Les embryons sont de bonne qualité, s'implantent facilement sans fausse-couche et les enfants sont normaux.

Si besoin est (femme mauvaise répondeuse), on répétera les tentatives d'ICSI.

\begin{tabular}{|c|c|c|c|}
\hline & Patient $A$ & Patient B & Patient C \\
\hline Age & 26 ans & 35 ans & 47 ans \\
\hline \multicolumn{4}{|l|}{ Avant thérapie } \\
\hline $\begin{array}{l}\text { Concentration des } \\
\text { spermatozoïdes }\end{array}$ & 0 & 0 & 0 \\
\hline Volume testiculaire & $5 \mathrm{ml} / 8 \mathrm{ml}$ & $4 \mathrm{ml} / 4 \mathrm{ml}$ & $5 \mathrm{ml} / 5 \mathrm{ml}$ \\
\hline $\begin{array}{l}\text { Valeurs sériques de FSH } \\
(\mathrm{IU} / \mathrm{L}) / \mathrm{LH}(\mathrm{IU} / \mathrm{L}) / \\
\text { testostérone }(\mathrm{nmol} / \mathrm{L})\end{array}$ & $0.23 / 0.09 / 0.6$ & $0.07 / \mathrm{ND}^{*} / \mathrm{ND}$ & $\begin{array}{l}0.07 /<0.01 / 60.3 \\
\text { (le patient est alors sous } \\
\text { traitement substitutif de } \\
\text { testostérone) }\end{array}$ \\
\hline \multicolumn{4}{|c|}{ Après 3 mois de traitement hormonal } \\
\hline $\begin{array}{l}\text { Concentration } \\
\text { spermatozoïdes }\end{array}$ & 0 & OAT III** & 0 \\
\hline $\begin{array}{l}\text { Etat de la spermatogénèse } \\
\text { (biopsie testiculaire) }\end{array}$ & & Hypoplasie germinale & Hypoplasie germinale \\
\hline Volume testiculaire & ND & $7 \mathrm{ml} / 7 \mathrm{ml}$ & $6 \mathrm{ml} / 8 \mathrm{ml}$ \\
\hline \multicolumn{4}{|c|}{ Après 6 mois de traitement hormonal } \\
\hline $\begin{array}{ll}\begin{array}{l}\text { Concentration } \\
\text { spermatozoïdes }\end{array} & \text { des } \\
\end{array}$ & OAT III & OAT III & 0 \\
\hline ICSI & $\begin{array}{l}\text { Utilisation de spermatozoïdes } \\
\text { éjaculés congelés ( } 14 \text { mois de } \\
\text { traitement). Grossesse à la } \\
\text { 4ème tentative. Naissance à } \\
\text { terme. }\end{array}$ & $\begin{array}{c}\text { Utilisation de spermatozoïdes } \\
\text { frais éjaculés. Grossesse à la } \\
\text { 4ème tentative ( } 23 \text { mois de } \\
\text { traitement). Naissance à } \\
\text { terme. }\end{array}$ & $\begin{array}{l}\text { Utilisation de spermatozoïdes } \\
\text { frais éjaculés. Grossesse à la } \\
\text { lère tentative ( } 11 \text { mois de } \\
\text { traitement). Naissance à } \\
\text { terme. }\end{array}$ \\
\hline
\end{tabular}

*ND : non déterminé. **OAT III : oligoasthénozoospermie avec $<5.10^{6}$ spermatozoïdes $/ \mathrm{ml},<5 \%$ de spermatozoïdes mobiles et $<5 \%$ de formes normales. 
Evaluation de la capacitation in vitro du spermatozoïde humain par le contenu en phosphotyrosines d'une protéine de 107KDA

\author{
S. Bréchard, C. Picherit, D. Boucher, G. Grizard \\ Laboratoire de Biologie du Développement et de la \\ Reproduction, Faculté de Médecine, place Henri \\ Dunant, 63000 Clermont-Fd,et CECOS, CHU, 63058 \\ Clermont-Fd, France
}

\section{Introduction :}

La capacitation du spermatozoïde humain, étape préalable indispensable à l'expression de son pouvoir fécondant, peut être induite in vitro en provoquant une fuite du cholestérol de la membrane plasmique vers le milieu extracellulaire, ce qui conduit à la phosphorylation des résidus tyrosines de certaines protéines (Osheroff et al., 1999)*. L'objectif de ce travail est d'évaluer la capacitation induite par des accepteurs de cholestérol (bovine serum albumin : BSA ; 2-hydroxy-propyl- $\beta$-cyclodextrine: $\beta$-CD), d'une part en utilisant la méthode classique (appréciation de la réaction acrosomique (RA) induite), et d'autre part en appréciant le niveau de phosphorylation des résidus tyrosines de protéines cellulaires.

\section{Matériel et Méthodes :}

L'étude est réalisée sur 10 spermes normaux (d'après les critères de l'OMS). Après migration sur gradient discontinu de densité (47,5\% - 95\% de Percoll), seuls les spermatozoïdes de la fraction $95 \%$ sont retenus. Cinq millions de spermatozoïdes sont incubés pendant 3 heures $\left(5 \% \mathrm{CO}_{2}\right.$; $\left.37^{\circ} \mathrm{C}\right)$ dans le milieu $\mathrm{BWW}$ seul, ou supplémenté en BSA à $3 \mathrm{mg} / \mathrm{ml}(+\mathrm{BSA})$ ou en $\beta-\mathrm{CD}$ à $0,5 \mathrm{mM}\left(+\beta-\mathrm{CD}_{0,5}\right), 1$ $\mathrm{mM}\left(+\beta-\mathrm{CD}_{1}\right)$ ou $2 \mathrm{mM}\left(+\beta-\mathrm{CD}_{2}\right)$, dans un volume final

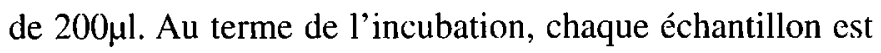
réparti en 2 aliquotes. Sur l'une, la RA est induite par l'ionophore calcique $\mathrm{A} 23187(10 \mu \mathrm{M} ; 45 \mathrm{~min})$ et un témoin sans ionophore est incubé dans les mêmes conditions. Les spermatozoïdes ayant effectué leur RA sont visualisés par immunofluorescence (marquage à l'agglutinine de Pisum sativum couplée au fluoresceine isothiocyanate). Les résultats sont exprimćs en \% de la totalité des cellules vivantes (test de gonflement hypo-osmotique). Sur l'autre aliquote, après Western blot des protéines et immunodétection de celles contenant des phosphotyrosines à l'aide de l'anticorps primairc Py69 (IgG de souris anti-phosphotyrosines; Interchim), une analyse semi-quantitative est réalisée par densitométrie. Les résultats sont exprimés par rapport à un standard interne.

\section{Résultats :}

Par comparaison au BWW, la supplémentation du milieu avec les accepteurs de cholestérol provoque une hausse significative de la capacité des spermatozoïdes à faire une RA induite (+BSA: $+61 \% ;+\beta-\mathrm{CD}_{0,5}:+61 \%$ ). Après capacitation des spermatozoïdes, 2 bandes protéiques (PM apparents: 75 et $107 \mathrm{kDa}$ ) sont majoritairement phosphorylées. La phosphorylation est prédominante et facilement quantifiable pour la protéine de $107 \mathrm{kDa}$, qui est donc seule prise en compte. Comparée au BWW, l'incubation en présence des accepteurs de cholestérol se traduit par une augmentation significative du contenu en phosphotyrosines de cette protéine $\left(+\mathrm{BSA}:+88 \% ;+\beta-\mathrm{CD}_{0,5}:+84 \%\right)$. En outre, dans nos conditions expérimentales, l'effet stimulateur de la $\beta$-CD (aussi bien sur la capacité des cellules à faire une RA induite que sur le niveau de phosphorylation des résidus tyrosines de la protéine de $107 \mathrm{kDa}$ ) augmente avec la concentration utilisée.

\section{Conclusion :}

Nos résultats montrent que le contenu en phosphotyrosines d'une protéine de $107 \mathrm{kDa}$ peut être utilisé comme marqueur moléculaire de la capacitation des spermatozoïdes humains.

* Osheroff et al. (1999) Molecular Human Reproduction 5: 1017-1026.

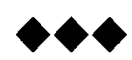

\section{Aneuploïdies spermatiques après traitement par radiothérapie et/ou chimiothérapie pour cancer testiculaire ou lymphome Etude de 13 patients}

\section{C.THOMAS 1, C.DE ROBERTIS2, R.PELLETIER2, S.ROUSSEAUX2, B.SELE 1,2, S.HENNEBICQ ${ }^{1,2}$ \\ 1 CECOS, Centre Hospitalo-universitaire de Greno- ble, BP 217, 38043 Grenoble Cedex 09, France \\ 2 NSERM U309, Institut Albert Bonniot, Université Joseph Fourier, Domaine de la Merci, 38706 Grenoble, France}

\section{Introduction :}

Les traitements anticancéreux ont fait de considérables progrès ces dernières années permettant ainsi d'améliorer 
le pronostic de nombreuses affections cancéreuses. Les effets secondaires de ces traitements sur la fonction de reproduction masculine sont de deux types : ils entraînent une inhibition de la spermatogenèse et sont susceptibles d'altérer le contenu génétique des cellules germinales. Parmi ces altérations génétiques, les anomalies chromosomiques pourraient, si elles persistent après reprise de la spermatogenèse, être responsables de fausse-couches spontanées ou de malformations congénitales.

Les effets à court terme (moins de 3 mois) sur le contenu chromosomique des spermatozoïdes sont maintenant assez bien évalués dans la littérature. Les cassures de l'ADN et les anomalies de ségrégation des chromosomes au cours de la méiose sont responsables d'anomalies structurales et numériques. En revanche, à plus long terme, les conséquences ne sont pour l'instant pas clairement établies : le nombre de patients inclus dans chaque étude est faible $(6$ au maximum), et les conclusions des analyses directes du contenu chromosomique des gamètes sont contradictoires.

\section{Matériel et Méthodes :}

Au CHU de Grenoble, nous avons comparé les taux d'aneuploïdies spermatiques de sujets ayant été traités par radiothérapie et/ou chimiothérapie entre 1995 et 2000 pour cancer du testicule ou pour lymphome avec ceux d'une population témoin de spermes de sujets fertiles sains.

\section{Résultats :}

L'analyse par FISH de la ségrégation des chromosomes $\mathrm{X}, \mathrm{Y}$ et 18 de 13 patients a montré pour 4 d'entre eux une augmentation significative des taux d'aneuploïdies (disomies XY et diploïdies essentiellement). Ces anomalies ne semblent pas être corrélées au délai post-thérapeutique, à la numération spermatique ou à la tératozoospermie.

Au total, les données de la littérature et les résultats préliminaires de notre étude montrent que les augmentations des taux d'aneuploïdies spermatiques à distance des traitements anticancéreux sont peu fréquentes et que ces taux sont variables d'un patient à l'autre sans que l'on retrouve de corrélation avec des paramètres clinico-biologiques.

\section{Conclusion :}

Ces résultats sont encore partiels, mais nous pouvons proposer 2 attitudes pratiques dans le cadre de l'aide médicale à la procréation. D'une part, il paraît raisonnable chez ces patients d'attendre au minimum 2 ans avant d'envisager une AMP avec sperme frais. D'autre part, lorsque se pose la question de l'utilisation de sperme frais ou congelé, une analyse du contenu chromosomique des gamètes par FISH pourrait être proposée afin de prendre la meilleure décision pour chaque patient dans son projet parental. 